\title{
DEFORMATIONS OF TRANSVERSELY SYMPLECTIC AND TRANSVERSELY CONTACT FOLIATIONS
}

\author{
By \\ J. Girbau and G. GuAsP*
}

\section{Introduction}

The aim of this paper is to give a versality theorem for deformations of transversely holomorphic foliations on a compact manifold with an additional transversely symplectic or transversely contact structure and give some examples of deformations of such structures.

First of all recall the definitions of (holomorphic) symplectic and contact manifolds. A complex manifold $M$ of even complex dimension $q=2 q^{\prime}$ admits a (holomorphic) symplectic structure if there exists a closed holomorphic 2-form $\omega$ such that $\omega^{q^{\prime}} \neq 0$ at each point. If the complex dimension $q$ of $M$ is odd, $q=$ $2 q^{\prime}+1$, and there is an atlas $\left\{\left(U_{i}, z_{i}^{1}, \cdots, z_{i}^{q}\right)\right\}$, a family $\left\{\omega_{i}\right\}$ of holomorphic 1forms on each $U_{i}$ such that $\omega_{i} \wedge\left(d \omega_{i}\right)^{q^{\prime}} \neq 0$ at each point and there are holomorphic functions $e_{i j}$ on $U_{i} \cap U_{j}$ with $\omega_{i}=e_{i j} \omega_{j}$ then $M$ is said to be a (holomorphic) contact manifold.

In 1960 Kodaira studied the theory of deformations of such structures ([7]) (among some other types of structures.) With the notations of that paper, the symplectic complex manifolds are the $\Gamma_{a}(\omega)$-structures with $\omega=d z^{1} \wedge d z^{2}+\cdots+$ $d z^{q-1} \wedge d z^{q}$ and the contact complex manifolds correspond to the $\Gamma_{r}(\omega)$-structures with $\omega=d z^{1}+z^{2} d z^{3}+\cdots+z^{q-1} d z^{q}$.

A transversely holomorphic foliation of complex codimension $q$ on a manifold $M$ can be defined as a $\Lambda^{t r}$-structure, where $\Lambda^{t r}$ denotes the pseudogroup of local diffeomorphisms of $\boldsymbol{R}^{p} \times \boldsymbol{C}^{q}$ of the form $f=\left(f^{u}, f^{a}\right)$ such that

$$
\frac{\partial f^{b}}{\partial x^{u}}=\frac{\partial f^{b}}{\partial \bar{z}^{a}}=0
$$

for $u \in\{1, \cdots, p\}$ and $a, b \in\{1, \cdots, q\}$. This means that $M$ is endowed with an atlas with local charts modeled in $\boldsymbol{R}^{p} \times \boldsymbol{C}^{q}$ whose coordinate changes belong to $A^{t r}$. The subbundle $F$ of ${ }^{c} T M$ locally generated by the vector fields of the

* Dep. de Matemàtiques, Univ. Autònoma de Barcelona. 08193 Bellaterra (Barcelona). Received November 5, 1990. 
form $\left(\partial / \partial x_{i}^{u}\right),\left(\partial / \partial \bar{z}_{i}^{a}\right)$ characterizes the foliation.

Let $\mathscr{I}$ be a transversely holomorphic foliation on a manifold $M$. If the complex codimension $q$ of $\mathscr{F}$ is even, $q=2 q^{\prime}$, we shall say that $\mathscr{I}$ admits a transversely (holomorphic) symplectic structure if there is a complex-valued 2form $\omega$ on $M$ such that:

1. $\omega(X, Y)=0$ if $X \in \Gamma(F)$.

2. $L_{X} \omega=0$ if $X \in \Gamma(F)$.

3. $\omega^{q^{\prime}} \neq 0$ at each point.

4. $d \omega=0$.

If the codimension $q$ of $\mathscr{F}$ is odd, $q=2 q^{\prime}+1$, we shall say that $\mathscr{F}$ admits a transversely (holomorphic) contact structure if there exists a complex-valued 1form $\omega_{U}$ on a neighbourdhood $U$ of each point such that

1. $\omega_{U}(X)=0$ if $X \in \Gamma(F)$.

2. $L_{X} \omega_{U}=0$ if $X \Subset \Gamma(F)$.

3. $\omega_{U} \wedge\left(d \omega_{U}\right)^{q^{\prime}} \neq 0$ at each point.

4. If $U \cap V \neq \varnothing$ one has $\omega_{U}=e_{U V} \omega_{V}$ on $U \cap V$, where $e_{U V}$ is a basic transversely holomorphic function, that is, $X\left(e_{U V}\right)=0$ if $X \in \Gamma(F)$.

From the point of view of the pseudogroups a manifold $M$ endowed with one of the two structures above is nothing but a $\Lambda_{\omega}^{t r}$-manifold, where $\Lambda_{\omega}^{t r}$ is the pseudogroup of local diffemorphisms of $\boldsymbol{R}^{p} \times \boldsymbol{C}^{q}$ of the form $f=\left(f^{u}, f^{a}\right)$, such that

$$
\frac{\partial f^{b}}{\partial x^{u}}=\frac{\partial f^{b}}{\partial \bar{z}^{a}}=0
$$

for $u \in\{1, \cdots, p\}$ and $a, b \in\{1, \cdots, q\}$, fullfilling $f^{*} \omega=\omega$ with $\omega=d z^{1} \wedge d z^{2}+\cdots$ $+d z^{q-1} \wedge d z^{q}$ in the symplectic case, and $f^{*} \omega=e(z) \omega$ with $e(z)$ a non-vanishing holomorphic function of $\boldsymbol{C}^{q}$ and $\omega=d z^{1}+z^{2} d z^{3}+\cdots+z^{q-1} d z^{q}$ in the contact case.

Let us give some examples of these structures. The complex projective spaces $\boldsymbol{C} \boldsymbol{P}^{2 n+1}$ of odd dimension are holomorphic contact manifolds in the following way ([6]). Let $U_{i}$ be the open set of $\boldsymbol{C P}^{2 n+1}$ of those points with homogeneous coordinates $z^{1}, \cdots, z^{2 n+2}$ such that $z^{i} \neq 0$. Let $s_{i}$ be the map

$$
\begin{gathered}
U_{i} \longrightarrow C^{2 n+2} \\
{\left[z^{1}, \cdots, z^{2 n+2}\right] \longrightarrow\left(z^{1} / z^{i}, \cdots, z^{i-1} / z^{i}, 1, z^{i+1} / z^{i}, \cdots, z^{2 n+2} / z^{i}\right)}
\end{gathered}
$$

where $\left[z^{1}, \cdots, z^{2 n+2}\right]$ means the point of homogenous coordinates $z^{1}, \cdots, z^{2 n+2}$. Let $\omega_{i}$ be the 1-form on $U_{i}$ given by $\omega_{i}=s_{i}^{*} \omega$, where $\omega$ is the following 1-form of $\boldsymbol{C}^{2 n+2}$

$$
\omega=\left(z^{1} d z^{2}-z^{2} d z^{1}\right)+\cdots+\left(z^{2 n+1} d z^{2 n+2}-z^{2 n+2} d z^{2 n+1}\right) .
$$


One has $\omega_{i}=e_{i j} \omega_{j}$ on $U_{i} \cap U_{j}$, with $e_{i j}=\left(z^{i} / z^{j}\right)^{2}$. By virtue of this construction the transversely holomorphic foliation on the real sphere $S^{4 n+3}$ given by the Hopf fibration $S^{4 n+3} \rightarrow \boldsymbol{C} \boldsymbol{P}^{2 n+1}$ is endowed with a natural transversely contact structure.

Another natural example is the projective co-tangent bundle $M$ of $\boldsymbol{C P}^{2 n+1}$ (that is, the projectivization of the co-tangent bundle of $\boldsymbol{C} \boldsymbol{P}^{2 n+1}$ ). As $\boldsymbol{C} \boldsymbol{P}^{2 n+1}$ admits a holomorphic contact structure, $M$ is endowed with the transversely contact structure associated to the foliation given by the bundle $M \rightarrow C \boldsymbol{P}^{2 n+1}$.

Since the complex torus $T^{2 n} \boldsymbol{C}$ obtained by the quotient $\boldsymbol{C}^{2 n} /(\boldsymbol{Z}+i \boldsymbol{Z})^{2 n}$ admits a natural holomorphic symplectic structure induced by the 2-form of $C^{2 n} \omega=$ $d z^{1} \wedge d z^{2}+\cdots+d z^{2 n-1} \wedge d z^{2 n}$, all the bundles $M \rightarrow T^{2 n} C$ induce transversely holomorphic foliations on $M$ with a transversely symplectic structure.

Another non-trivial example of such structures is the suspension of an isomorphism of the symplectic structure of $T^{2 n} \boldsymbol{C}$ constructed in the following way. Given $A \in \mathrm{Sp}(2 n, \boldsymbol{Z})$, take the quotient manifold $M$ of $\boldsymbol{R} \times T^{2 n} \boldsymbol{C}$ by the equivalence relation identifying $(t, z)$ with $(t+1, A(z))$. Take the transversely holomorphic foliation $\mathscr{I}$ on $M$ whose leaves are induced by the lines $\boldsymbol{R} \times\{z\}$. As $A$ preserves $\omega$ then $\mathscr{F}$ is transversely symplectic.

The theory of deformations of holomorphic foliations was initiated by Kodaira and Spencer [8] in 1961. Gómez-Mont [5] and Duchamp-Kalka [2] gave a weak version of the versality theorem (often called Kuranishi's theorem) for deformations of a transversely holomorphic foliation on a compact manifold. Girbau, Haefliger and Sundaraman [4], using the original ideas of Kodaira and Spencer as well as a version of the classical Kuranishi's theorem for complex structures given by Douady [1], obtained a strong versality theorem for deformations of transversely holomorphic foliations.

One of the aims of this paper is to give such a versality theorem for deformations of transversely holomorphic foliations endowed with an additional transversely (holomorphic) symplectic or contact structure. The sheaf of infinitesimal transformations of these stuctures is the sheaf $\Theta_{\omega}^{t r}$ of germs of $C^{\infty}$ local vector fields $X$ whose expression in a local chart $\left(U_{i}, x_{i}^{u}, z_{i}^{a}\right)$ adapted to the foliation is

$$
X=\sum X^{a}\left(z_{i}\right) \frac{\partial}{\partial z_{i}^{a}}+X^{u}\left(x_{i}, z_{i}, \bar{z}_{i}\right) \frac{\partial}{\partial x_{i}^{u}},
$$

where the $X^{a}$ are holomorphic functions and $X$ fulfils, moreover, the condition $L_{X} \omega=0$ in the symplectic case or $L_{X} \omega_{i}=\lambda_{i} \omega_{i}$ in the contact case, where $\lambda_{i}$ is a basic transversely holomorphic function. 
As Kodaira and Spencer say in the introduction of their "Multifoliate structures" [8], the following pattern is needed to study the deformations of a given structure :

1. A fine resolution of the sheaf of germs of infinitesimal transformations of the structure such that its space of sections is an elliptic complex.

2. A Lie algebra structure in this resolution compatible with its differential operator.

3. A procedure associating a family of deformations of the structure to each family of degree 1 sections of the resolution, fulfilling a suitable integrability condition which depends on the Lie algebra product of point 2 .

The fine resolutions of $\Theta_{\omega}^{t r}$ we give here (point 1) (in the symplectic as well as the contact cases) are a combination of the resolutions of "Multifoliate structures" and those given by Kodaira in [7]. Once the above plan is accomplished we are able to prove a versality theorem for these structures using the same construction that in [4].

In this paper we put special emphasis on computing the versal space and the versal family of deformations for the natural examples described above. For the Hopf fibration $S^{4 n+3} \rightarrow \boldsymbol{C} \boldsymbol{P}^{2 n+1}$ with its transversely contact structure we prove that the versal space is smooth, that is, a neighbourhood of the origin in the vector space $H^{1}\left(S^{4 n+3}, \Theta_{\omega}^{t r}\right) \cong H^{0}\left(\boldsymbol{C} \boldsymbol{P}^{2 n+1}, \Theta_{\omega}\right)$, where $\Theta_{\omega}$ is the sheaf of germs of holomorphic vector fields $X$ on $C P^{2 n+1}$ fulfilling $L_{X} \omega_{i}=\lambda_{i} \omega_{i}$. This is a vector space of complex dimension $(2 n+2)^{2}-(1+2+\cdots+(2 n+1))$. Remark that the versal space of the same foliation without the contact structure $[3]$, [4]) is also smooth and its dimension is $(2 n+2)^{2}-1$.

For the projectivization of the contangent bundle of $\boldsymbol{C} \boldsymbol{P}^{2 n+1}$ with its natural transversely contact structure we find that this structure is rigid.

An example of transversely contact structure with non-smooth versal space is the product $T^{n} \boldsymbol{R} \times \boldsymbol{C} \boldsymbol{P}^{3}$ with the trivial transversely holomorphic foliation whose leaves are $T^{n} \boldsymbol{R} \times\{p\}$.

For the transversely symplectic structures described above we obtain the following results. When $M$ is the quotient manifold $\boldsymbol{R} \times T^{2 n} \boldsymbol{C}$ by the equivalence relation $(t, z) \sim(t+1, A(z))$ with $A \in \mathrm{Sp}(2 n, Z)$ we find that the versal space is smooth and its dimension is the sum of dimensions of the three vector spaces $H^{0}\left(T^{2 n} \boldsymbol{C}, \Theta_{A}\right), P_{A}$ and $Q_{A}$, where $H^{0}\left(T^{2 n} C, \Theta_{A}\right)$ is the space of holomorphic vector fields on $T^{2 n} C$ invariant by $A, P_{A}$ is the space of those $2 n \times 2 n$ matrices commuting with $A$ and $Q_{A}$ is the vector space of those $2 n \times 2 n$ antisymmetric matrices $\varepsilon$ such that ${ }^{t} A \varepsilon A=A$. For example, if $n=1$ and 


$$
A=\left(\begin{array}{ll}
a & b \\
c & d
\end{array}\right)
$$

then the versal space has dimension 3 when $a+d \neq 2$ and dimension 4 when $a+d=2$.

Finally we give an example of transversely symplectic structure with nonsmooth versal space. For this purpose we take a $S^{1}$-principal bundle over the torus $T^{2 n} \boldsymbol{C}=\boldsymbol{C}^{2 n} /(\boldsymbol{Z}+i \boldsymbol{Z})^{2 n}, M \rightarrow T^{2 n} \boldsymbol{C}$, with Euler class $e(M) \in H^{1,1}\left(T^{2 n} \boldsymbol{C}\right)$. In this case the space of infinitesimal deformations, $H^{11}\left(M, \Theta_{\omega}^{t r}\right)$, has dimension $(2 n)^{2}+\left(\begin{array}{c}2 n \\ 2\end{array}\right)+\operatorname{dim} E_{\Omega}$, where $E_{\Omega}$ is the space of holomorphic vector fields $X$ on $T^{2 n} \boldsymbol{C}$ fulfilling $\Omega \wedge i_{X} \omega=0$, where $\omega$ is the symplectic form of $T^{2 n} \boldsymbol{C}$ and $\Omega$ is the harmonic representative of Euler class (that is, a 2-form). So the dimension of $H^{1}\left(M, \Theta_{\omega}^{t r}\right)$ depends on $\Omega$. We compute the versal space giving its equation and showing that for many 2 -forms $\Omega$ this space is not smooth.

\section{Resolutions of the sheaves $\Theta_{\omega}^{t r}$}

Let $I$ be a transversely holomorphic foliation on a manifold $M$ endowed with an additional transversely contact or symplectic structure. Let $\hat{\Theta}_{\omega}^{t r}$ be the sheaf of germs of $\mathcal{C}^{\infty}$ local vector fields $X$ which in local coordinates $\left(x^{u}, z^{a}\right)$ adapted to $\mathscr{F}$ are of the form

$$
X=\sum X^{a}(z) \frac{\partial}{\partial z^{a}}+X^{\bar{a}} \frac{\partial}{\partial \bar{z}^{a}}+X^{u} \frac{\partial}{\partial x^{u}},
$$

where the components $X^{a}$ are transversely holomorphic functions and $X$ fulfils, moreover, the condition $L_{X} \omega=0$ in the symplectic case and $L_{X} \omega_{i}=\lambda_{i} \omega_{i}$ in the contact case, where $\lambda_{i}$ are transversely holomorphic functions. We shall give a resolution of $\hat{\Theta}_{\omega}^{t r}$ instead of the sheaf $\Theta_{\omega}^{t r}$ defined in the introduction. Remark that $\Theta_{\omega}^{t r}$ is the quotient of $\hat{\Theta}_{\omega}^{t r}$ by a fine subsheaf, thus the cohomology of $M$ with values in these two sheaves is the same, except in degree 0 .

Let $A^{*}(M)$ be the graded Lie algebra of $C^{\infty}$ complex-valued differential forms on $M$. Kodaira and Spencer [8] show that every degree $k$ derivation $\delta$ of $A^{*}(M)$ is determined in a local chart $\left(x^{u}, z^{a}\right)$ by a couple $(\varphi, \xi)$ of vector forms of degrees $k$ and $k+1$,

$$
\begin{aligned}
& \varphi=\Sigma \varphi^{u} \frac{\partial}{\partial x^{u}}+\varphi^{a} \frac{\partial}{\partial z^{a}}+\varphi^{\bar{a}} \frac{\partial}{\partial \bar{z}^{a}} \\
& \xi=\Sigma \xi^{u} \frac{\partial}{\partial x^{u}}+\xi^{a} \frac{\partial}{\partial z^{a}}+\xi^{\bar{a}} \frac{\partial}{\partial \bar{z}^{a}}
\end{aligned}
$$


where $\varphi^{u}=\delta\left(x^{u}\right), \varphi^{a}=\delta\left(z^{a}\right), \varphi^{\bar{a}}=\delta\left(\bar{z}^{a}\right), \xi^{u}=(-1)^{k} \delta\left(d x^{u}\right), \xi^{a}=(-1)^{k} \delta\left(d z^{a}\right), \xi^{\bar{a}}=$ $(-1)^{k} \delta\left(d \bar{z}^{a}\right)$. The vector $k$-forms $\varphi_{i}$ on each local chart $U_{i}$ glue together giving a global vector $k$-form. If we denote by $d \varphi_{i}$ the vector $(k+1)$-form on $U_{i}$ given by

$$
d \varphi_{i}=\Sigma d \varphi_{i}^{u} \frac{\partial}{\partial x_{i}^{u}}+d \varphi_{i}^{a} \frac{\partial}{\partial z_{i}^{a}}+d \varphi_{i}^{\bar{a}} \frac{\partial}{\partial \bar{z}_{i}^{a}}
$$

then the $d \varphi_{i}-\xi_{i}$ give a global vector $(k+1)$-form denoted by $d \varphi-\xi$. Denote by $\eta$ the vector $(k+1)$-form $\eta=(-1)^{k}(\xi-d \varphi)$. Then the derivation $\delta$ is determined by the couple $(\varphi, \eta)$. If $\sigma \in A^{r}(M)$ then the action of $\delta$ on $\sigma$ is given in terms of $\varphi$ and $\eta$ by the following expression :

$$
\delta(\sigma)=(-1)^{k} d(\varphi \bar{\wedge} \sigma)+\varphi \bar{\wedge} d \sigma+\eta \bar{\wedge} \sigma,
$$

where the product $\bar{\wedge}$ is defined in [8]. Recall that $(\alpha \otimes X) \wedge \sigma=\alpha \wedge i_{X} \sigma$ when $\alpha$ and $\sigma$ are ordinary differential forms and $X$ is vector field.

Denote by $\mathscr{D}_{0}^{k}$ the vector space of all the derivations of degree $k$ of $A^{*}(M)$. $\mathscr{D}_{0}^{k}$ is endowed with a natural Lie bracket defined by $\left[\delta, \delta^{\prime}\right]=\delta \delta^{\prime}-(-1)^{k k^{\prime}} \delta^{\prime} \delta$, where $k$ and $k^{\prime}$ are the respective degrees of $\delta$ and $\delta^{\prime}$. If $\delta=(\varphi, \eta)$ and $\delta^{\prime}=$ $\left(\varphi^{\prime}, \eta^{\prime}\right)$ then

$$
\left[\delta, \delta^{\prime}\right]=\left(\left[\varphi, \varphi^{\prime}\right]+\eta^{\prime} \bar{\wedge} \varphi+\eta \bar{\wedge} \varphi^{\prime},-\left[\eta, \varphi^{\prime}\right]-\left[\eta^{\prime}, \varphi\right]+\eta^{\prime} \bar{\wedge} \eta+\eta \bar{\wedge} \eta^{\prime}\right),
$$

where the brackets in the right hand side are those of vector forms and the product $\bar{\wedge}$ means here the product of vector forms fulfilling $\varphi \bar{\wedge}(\beta \otimes Y)=(\varphi \bar{\wedge} \beta)$ $\otimes Y$, where $\beta$ is an ordinary form.

We have a differential $D: \mathscr{D}_{0}^{k} \rightarrow \mathscr{D}_{0}^{k+1}$ defined by $D(\delta)=[d, \delta]$, where $d$ is the exterior derivative. If $\delta=(\varphi, \eta)$ then $D \delta=\left((-1)^{k} \eta, 0\right)$.

Let $I_{F}$ be the ideal of $A^{*}(M)$ of those forms $\omega$ such that $\omega\left(X_{1}, \cdots, X_{k}\right)=0$ when $X_{1}, \cdots, X_{k}$ are sections of $F$. (Recall that $F$ is the subbundle of ${ }^{c} T M$ locally generated by $\left.\left\{\left(\partial / \partial x^{u}\right),\left(\partial / \partial \bar{z}^{a}\right)\right\}.\right) \quad I_{F}$ is locally generated by $\left\{d z^{a}\right\}$. Let $\mathscr{D}^{k}$ be the subspace of $\mathscr{D}_{0}^{k}$ of those derivations $\delta$ such that $\delta\left(I_{F}\right) \subset I_{F}$. Since $d\left(I_{F}\right) \subset I_{F}, D$ maps $\mathscr{D}^{k}$ into $\mathscr{D}^{k+1}$.

\subsection{The symplectic case}

Denote by $S_{(2)}^{k}$ the subbundle of $\Lambda^{k}\left({ }^{c} T M\right)^{*}$ of those $\sigma$ which can be written in local coordinates

$$
\sigma=\sum \sigma_{a b} \wedge d z^{a} \wedge d z^{b}
$$

with $\sigma_{a b} \in \Lambda^{k-2}\left({ }^{c} T M\right)^{*}$. Let $\mathcal{S}_{(2)}^{k}$ be the sheaf of germs of sections of $S_{(2)}^{k}$. Denote by $\widetilde{D}^{k}$ the sheaf of germs of elements of $\mathscr{D}^{k}$. Set $\Phi_{\omega}^{0}=\widetilde{D}_{0}$ and $\Phi_{\omega}^{k}=$ $\widetilde{\mathfrak{D}}^{k} \oplus \mathcal{S}_{(2)}^{k+1}$ when $k \geqq 1$. Define the following maps : 


$$
\begin{gathered}
\iota: \hat{\Theta}_{\omega}^{t r} \longrightarrow \Phi_{\omega}^{0}=\widetilde{D}^{0} \quad \text { by } \quad \iota(X)=L_{X} \\
D_{\omega}: \Phi_{\omega}^{0} \longrightarrow \Phi_{\omega}^{1}=\widetilde{\mathscr{D}}^{1} \oplus \mathcal{S}_{(2)}^{2} \quad \text { by } \quad D_{\omega}(\delta)=(D \delta, \delta(\omega)) \\
D_{\omega}: \Phi_{\omega}^{k} \longrightarrow \Phi_{\omega}^{k+1} \text { when } k>0 \quad \text { by } \quad D_{\omega}(\delta, \sigma)=(D \delta, \delta(\omega)-d \sigma)
\end{gathered}
$$

In this way we have a sequence

$$
0 \longrightarrow \hat{\Theta}_{\omega}^{t r} \stackrel{\iota}{\longrightarrow} \Phi_{\omega}^{0} \stackrel{D_{\omega}}{\longrightarrow} \Phi_{\omega}^{1} \stackrel{D_{\omega}}{\longrightarrow} \cdots
$$

Let us prove its exactness. In fact a simple computation shows that $D_{\omega}^{2}=0$. To prove the exactness at $\Phi_{\omega}^{k}$ when $k \neq 0$ let $s=(\delta, \sigma)$ be such that $D_{\omega} s=0$. This is equivalent to the fact

$$
\left\{\begin{array}{l}
D \delta=0 \\
\delta(\omega)-d \sigma=0
\end{array}\right.
$$

From the first equation one sees that there exist $\delta^{\prime}$ with $D \delta^{\prime}=\delta$. Then second equation is now $d\left(\delta^{\prime}(\omega)-\sigma\right)=0$, so there is a section $\sigma^{\prime}$ of $\mathcal{S}_{(2)}^{k}$ with $\delta^{\prime}(\omega)-\sigma=$ $d \sigma^{\prime}$. Then

$$
D_{\omega}\left(\delta^{\prime}, \sigma^{\prime}\right)=\left(D \delta^{\prime}, \delta^{\prime}(\omega)-d \sigma^{\prime}\right)=(\delta, \sigma) .
$$

An analogous argument shows the exactness of (3) at $\Phi_{\omega}^{0}$.

\subsection{The contact case}

Suppose now that the contact structure is defined by a family $\left\{\omega_{i}\right\}$ of 1 forms on each local chart $U_{i}$ in such a way that $\omega_{i}=e_{i j} \omega_{j}$ on $U_{i} \cap U_{j}$. Let $A_{F}^{*}$ be the quotient $A^{*}(M) / I_{F}$. Let $E$ be the line bundle over $M$ defined by the transition functions $\left\{e_{i j}\right\}$. Let $\delta$ be a degree $k$ derivation of $A^{*}(M)$ and $\left\{\gamma_{i}\right\}$ a family of elements $\gamma_{i} \in A_{F}^{k}\left(U_{i}\right)$. We shall say that the couple $\left(\delta,\left\{\gamma_{i}\right\}\right)$ is an $E$-derivation if $\left[\delta\left(e_{i j}\right)\right]=e_{i j}\left(\gamma_{i}-\gamma_{j}\right)$ on $U_{i} \cap U_{j}$, where $\left[\delta\left(e_{i j}\right)\right]$ means the class of $\delta\left(e_{i j}\right)$ modulo $I_{F}$. We shall denote by $\mathscr{D}^{k}{ }_{E} A_{F}^{k}$ the space of $E$-derivations of degree $k$. As $d\left(I_{F}\right) \subset l_{F}$ the exterior derivative $d$ induces a differential $d_{F}$ on the quotient $A_{F}^{*}$. If $\left(\delta,\left\{\gamma_{i}\right\}\right)$ is an $E$-derivation then $\left(D \delta,\left\{d_{F} \gamma_{i}\right\}\right)$ is also an $E$ derivation. Let $S_{(1)}^{k}$ be the subbundle of $\Lambda^{k}\left({ }^{c} T M\right)^{*}$ of those $\sigma$ which in an adapted local chart $\left(x^{u}, z^{a}\right)$ are expressed by $\sigma=\Sigma \sigma_{a} \wedge d z^{a}$. Denote by $S_{(1) F}^{k}$ the quotient bundle $S_{(1)}^{k} / S_{(2)}^{k}$, where $S_{(2)}^{k}$ has been defined in the subsection 1.1 (the symplectic case). We can think of sections of $S_{(1) F}^{k}$ as families $\left\{\sigma_{i}\right\}$ of sections of $S_{(1)}^{k} \mid U_{i}$ such that $\sigma_{i}-\sigma_{j}$ is a section of $S_{(2)}^{k}$ on $U_{i} \cap U_{j}$. Denote by $S_{(1) F}^{k}(E)$ the vector bundle $S_{(1) F}^{k} \otimes E$. The sections of $S_{(1) F}^{k}(E)$ are families $\left\{\sigma_{i}\right\}$ of sections of $\left.S_{(1) F}^{k}\right|_{U_{i}}$ such that $\sigma_{i}=e_{i j} \sigma_{j}$ on $U_{i} \cap U_{j}$. Denote by $\widetilde{D}^{k}+_{E} \tilde{A}_{F}^{k}$ the sheaf of germs of $E$-derivations and by $\mathcal{S}_{(1) F}^{k}(E)$ the sheaf of germs of sections 
of $S_{(1) F}^{k}(E)$. Set $\Phi_{\omega}^{k}=\left(\widetilde{D}^{k}+{ }_{E} \tilde{A}_{F}^{k}\right) \oplus \mathcal{S}_{(1) F}^{k}(E)$. As $S_{(1) F}^{0}$ vanishes then $\Phi_{\omega}^{0}=\widetilde{D}^{0}+$ ${ }_{E} A_{F}^{0}$.

In order to define the differential $D_{\omega}: \Phi_{\omega}^{k} \rightarrow \Phi_{\omega}^{k+1}$ remark that the exterior differencial $d$ induces a differential $d_{S}$ in the sections of $S_{(1) F}^{k}$ (since $d\left(I_{F}\right) \subset I_{F}$ ). $d_{S}$ can be extended to sections of $S_{(1) F}^{k}(E)$ since $d e_{i j} \in I_{F}$ and if $\left\{\sigma_{i}\right\}$ is a section of $S_{(1) F}^{k}(E)$ then $d_{S} \sigma_{i}=e_{i j} d_{S} \sigma_{j}$. We define now $D_{\omega}: \Phi_{\omega}^{k} \rightarrow \Phi_{\omega}^{k+1}$ by

$$
D_{\omega}\left(\left(\delta,\left\{\gamma_{i}\right\}\right),\left\{\sigma_{i}\right\}\right)=\left(\left(D \delta,\left\{d_{F} \gamma_{i}\right\}\right),\left\{\left[\delta \omega_{i}\right]-\gamma_{i} \wedge\left[\omega_{i}\right]-d_{S} \sigma_{i}\right\}\right),
$$

where [ ] means here classes modulo $S_{(2)}^{k}$. Remark that $\left[\delta \omega_{i}\right]-\gamma_{i} \wedge\left[\omega_{i}\right]$ defines a section of $S_{(1) F}^{k+1}(E)$. In fact, as $\omega_{i}=e_{i j} \omega_{j}$ we have $\delta\left(\omega_{i}\right)=\delta\left(e_{i j}\right) \wedge \omega_{j}+e_{i j} \delta\left(\omega_{j}\right)$ and $\left[\delta\left(\omega_{j}\right)\right]=\left[\delta\left(e_{i j}\right)\right] \wedge\left[\omega_{j}\right]+e_{i j}\left[\delta\left(\omega_{j}\right)\right]$, where $\left[\delta\left(e_{i j}\right)\right]$ means the class of $\delta\left(e_{i j}\right)$ modulo $I_{F}$ and $\left[\omega_{i}\right]$ means the class of $\omega_{i}$ modulo $S_{(2)}^{k}$. So $\left[\delta\left(\omega_{i}\right)\right]=e_{i j}\left(\gamma_{i}-\gamma_{j}\right)$ $\wedge\left[\omega_{j}\right]+e_{i j}\left[\omega_{j}\right]$. That is

$$
\left[\delta\left(\omega_{i}\right)\right]-\gamma_{i} \wedge\left[\omega_{i}\right]=e_{i j}\left(\left[\delta\left(\omega_{j}\right)\right]-\gamma_{j} \wedge\left[\omega_{j}\right]\right) .
$$

Define the map $\iota: \hat{\Theta}_{\omega}^{t r} \rightarrow \Phi_{\omega}^{0}$ by $\iota(X)=\left(L_{X},\left\{\lambda_{i}(X)\right\}\right)$, where $\lambda_{i}(X)$ are those functions appearing in the expression $L_{X} \omega_{i}=\lambda_{i}(X) \omega_{i}$. In this way we have the sequence

$$
0 \longrightarrow \hat{\Theta}_{\omega}^{t r} \stackrel{\iota}{\longrightarrow} \Phi_{\omega}^{0} \stackrel{D_{\omega}}{\longrightarrow} \Phi_{\omega}^{1} \stackrel{D_{\omega}}{\longrightarrow} \cdots
$$

Let us prove its exactness. A straightforward computation shows that $D_{\omega}^{2}=0$. Suppose now $k \geqq 2$ and let $s$ be an element of $\Phi_{\omega}^{k}, s=\left(\left(\delta,\left\{\gamma_{i}\right\}\right),\left\{\sigma_{i}\right\}\right)$, with $D_{\omega} s=0$. This means

$$
\left\{\begin{array}{l}
D \delta=0 \\
d_{F} \gamma_{i}=0 \\
{\left[\delta\left(\omega_{i}\right)\right]-\gamma_{i} \wedge\left[\omega_{i}\right]-d_{S} \sigma_{i}=0}
\end{array}\right.
$$

Since $D \delta=0$ there is $\delta^{\prime}$ such that $D \delta^{\prime}=\delta$. Since $d_{F} \gamma_{i}=0$ there is $\lambda_{i}$ with $d_{F} \lambda_{i}$ $=\gamma_{i}$. Finally, the last equation of (5) is $\left[\left(D \delta^{\prime}\right)\left(\omega_{i}\right)\right]-d_{F} \lambda_{i} \wedge\left[\omega_{i}\right]-d_{S} \sigma_{i}=0$. Since $\omega_{i} \in I_{F}$ we shall have $\left[\left(D \delta^{\prime}\right)\left(\omega_{i}\right)\right]=\left[d \delta^{\prime}\left(\omega_{i}\right)\right]=d_{S}\left[\delta^{\prime} \omega_{i}\right]$. On the other hand $d_{F} \lambda_{i} \wedge\left[\omega_{i}\right]=d_{S}\left(\lambda_{i} \wedge\left[\omega_{i}\right]\right)$. So

$$
d_{S}\left(\left[\delta^{\prime} \omega_{i}\right]-\lambda_{i} \wedge\left[\omega_{i}\right]-\sigma_{i}\right)=0 .
$$

We can find a representative $\mu_{i}$ of $\left[\delta^{\prime} \omega_{i}\right]-\lambda_{i} \wedge\left[\omega_{i}\right]-\sigma_{i}$ with $\mu_{i} \in I_{F}$ and such that its exterior derivative with respect to the coordinates $x, \bar{z}$ vanishes. So we can find $\tau_{i} \in I_{F}$ such that its exterior derivative with respect to the coordinates $x, \bar{z}$ is $\mu_{i}$. We shall have

$$
D_{\omega}\left(\left(\delta^{\prime},\left\{\gamma_{i}\right\}\right),\left\{\left[\tau_{i}\right]\right\}\right)=\left(\left(\delta,\left\{\gamma_{i}\right\}\right),\left\{\sigma_{i}\right\}\right) .
$$


A similar argument applies when $k=1$ and $k=0$.

\subsection{The cohomology spaces $H^{k}\left(M, \hat{\Theta}_{\omega}^{t r}\right)$}

Suppose now that $M$ is compact. Since the resolutions (3) and (4) of $\hat{\Theta}_{\omega}^{t r}$ are fine we have $H^{k}\left(M, \hat{\Theta}_{\omega}^{t r}\right)=Z^{k} / B^{k}$, where $Z^{k}$ is the space of global sections $s$ of $\Phi_{\omega}^{k}$ such that $D_{\omega} s=0$ and $B^{k}$ the subspace of those sections of the form $D_{\omega} s^{\prime}$ with $s^{\prime}$ a section of $\Phi_{\omega}^{k-1}$. In the symplectic case the leading part of $D_{\omega}$ is

$$
(\delta, \sigma) \longmapsto(D \delta,-d \sigma) .
$$

In the contact case the leading part of $D_{\omega}$ is

$$
\left(\left(\delta,\left\{\gamma_{i}\right\}\right),\left\{\sigma_{i}\right\}\right) \longmapsto\left(\left(D \delta,\left\{d_{F} \gamma_{i}\right\}\right),\left\{d_{S} \sigma_{i}\right\}\right) .
$$

In both cases the ellipticity of the de Rham complex gives the ellipticity of the complex of sections of (3) and (4). So $H^{k}\left(M, \hat{\Theta}_{\omega}^{t r}\right)$ are finite dimensional for $k>0$.

\section{The bracket of sections of $\Phi_{\omega}^{*}$}

Let $s$ and $s^{\prime}$ be two sections af $\Phi_{\omega}^{k}$ and $\Phi_{\omega}^{l}$ respectively. We are going to define the bracket $\left[s, s^{\prime}\right]$ as a section of $\Phi_{\omega}^{k+l}$ in the following way. Suppose first we are in the symplectic case. Then $s=(\delta, \sigma)$ and $s^{\prime}=\left(\delta^{\prime}, \sigma^{\prime}\right)$ with $\delta \in \mathscr{D}^{k}$, $\delta^{\prime} \in \mathscr{D}^{l}, \sigma \in \Gamma\left(\mathcal{S}_{(2)}^{k+1}\right)$ and $\sigma^{\prime} \in \Gamma\left(\mathcal{S}_{(2)}^{l+1}\right)$. We define

$$
\left[s, s^{\prime}\right]=\left(\left[\delta, \delta^{\prime}\right],(-1)^{k} \delta\left(\sigma^{\prime}\right)-(-1)^{k} \delta^{\prime}(\sigma)\right) .
$$

In the contact case we shall have $s=\left(\left(\delta,\left\{\gamma_{i}\right\}\right),\left\{\sigma_{i}\right\}\right), s^{\prime}=\left(\left(\delta^{\prime},\left\{\gamma_{i}^{\prime}\right\}\right),\left\{\sigma_{i}^{\prime}\right\}\right)$. We define

$$
\begin{aligned}
{\left[s, s^{\prime}\right]=} & \left(\left(\left[\delta, \delta^{\prime}\right],\left\{\left[\delta\left(\tilde{\gamma}_{i}^{\prime}\right)-(-1)^{k l} \delta^{\prime}\left(\tilde{\gamma}_{i}\right)\right]\right),\right.\right. \\
& \left.\left\{\left[(-1)^{k}\left(\delta\left(\tilde{\sigma}_{i}^{\prime}\right)-\tilde{\gamma}_{i} \wedge \sigma_{i}^{\prime}\right)-(-1)^{k l+l}\left(\delta^{\prime}\left(\tilde{\sigma}_{i}\right)-\tilde{\gamma}_{i}^{\prime} \wedge \tilde{\sigma}_{i}\right)\right]\right\}\right),
\end{aligned}
$$

where $\tilde{\gamma}_{i}, \tilde{\sigma}_{i}, \tilde{\gamma}_{i}^{\prime}$ and $\tilde{\sigma}_{i}^{\prime}$ are representatives of $\gamma_{i}, \sigma_{i}, \gamma_{i}^{\prime}$ and $\sigma_{i}^{\prime}$ respectively.

If $s \in \Gamma\left(\Phi_{\omega}^{k}\right), s^{\prime} \in \Gamma\left(\Phi_{\omega}^{l}\right)$ and $s^{\prime \prime} \in \Gamma\left(\Phi_{\omega}^{m}\right)$ then one can prove the following facts :

1. $\left[s, s^{\prime}\right]=(-1)^{k l+1}\left[s^{\prime}, s\right]$.

2. $(-1)^{k m}\left[s,\left[s^{\prime}, s^{\prime \prime}\right]\right]+(-1)^{k l}\left[s^{\prime},\left[s^{\prime \prime}, s\right]\right]+(-1)^{m l}\left[s^{\prime \prime},\left[s, s^{\prime}\right]\right]=0$.

3. $D_{\omega}\left[s, s^{\prime}\right]=\left[D_{\omega} s, s^{\prime}\right]+(-1)^{k}\left[s, D_{\omega} s^{\prime}\right]$.

\section{The integrability condition}

\subsection{The symplectic case}

Fix $(\mathscr{I}, \omega)$ a transversely holomorphic foliation on a compact manifold $M$ 
with a transversely symplectic structure $\omega$. Denote by $F$ its associated bundle (defined in the introduction). We are going to associate a section of $\Phi_{\omega}^{1}$ to each couple $\left(\mathscr{F}^{\prime}, \omega^{\prime}\right)$, where $\mathscr{F}^{\prime}$ is a transversely holomorphic foliation close enough to $\mathscr{F}$ and $\omega^{\prime}$ a transversely symplectic structure on $\mathscr{F}^{\prime}$ close enough to $\omega$. Here close enough will mean that $\left(\mathscr{F}^{\prime}, \boldsymbol{\omega}^{\prime}\right)$ is such that all the steps in the construction we are going to give make sense.

Denote by $N^{1,0}={ }^{c} T M / F$ the normal bundle of type $(1,0)$ of $\mathscr{F}$. Choose a splitting ${ }^{c} T M=F \oplus N^{1,0}$. Let $F^{\prime}$ be the bundle corresponding to $\mathscr{F}^{\prime}$. Suppose that $F^{\prime}$ is close enough to $F$ in such a way that $F^{\prime}$ is the graphic of a morphism $\theta: F \rightarrow N^{1,0}$ of vector bundles. That is $F^{\prime}=\{X+\theta(X)$ with $X \in F\} . \theta$ can be extended to a morphism ${ }^{c} T M \rightarrow^{c} T M$ with the condition $\left.\theta\right|_{N 1,0}=0$. Let $\varphi$ be the vector form on $M$ defined by $\varphi=\mathrm{id}+\theta$. In each local chart $\left(U, x^{u}, z^{a}\right)$ of $M$ adapted to $\mathscr{F}$ set $\varphi_{u}=\varphi\left(\partial / \partial x^{u}\right), \varphi_{a}=\varphi\left(\partial / \partial z^{a}\right), \varphi_{a}=\varphi\left(\partial / \partial \bar{z}^{a}\right)$. Suppose that $\varphi$ is close enough to the identity in order that $\varphi_{u}, \varphi_{a}, \varphi_{\bar{a}}$ is a basis of ${ }^{c} T_{x} M$ at each point. We shall have $\left[\varphi_{\lambda}, \varphi_{\mu}\right]=\Sigma C_{\lambda \mu}^{\nu} \varphi_{\nu}$, where the indices $\lambda, \mu, \nu$ denote all the indices $a, \bar{a}$ and $u$. Let $\delta$ be the derivation whose first component is $\varphi$ and whose action on $d x^{\lambda}$ is

$$
\delta\left(d x^{\lambda}\right)=-\frac{1}{2} \sum C_{\nu \mu}^{\lambda} d x^{\nu} \wedge d x^{\mu},
$$

where $x^{\lambda}$ means $z^{a}$ or $\bar{z}^{a}$ when $\lambda=a$ or $\lambda=\bar{\alpha}$.

As $F^{\prime}$ is an integrable distribution (it corresponds to the foliation $\mathscr{F}^{\prime}$ ) one has $C_{\alpha \beta}^{a}=0$ when $\alpha, \beta \in\{u, \bar{a}\}$. So $\delta \in \mathscr{D}^{1}$. $\delta$ fulfils $[\delta, \delta]=0$ (see [8]). Define $\tilde{\delta}=d-\delta$. Then the condition $[\delta, \delta]=0$ is equivalent to $D \tilde{\delta}-(1 / 2)[\tilde{\delta}, \tilde{\delta}]=0$. Define the 2-form $\varepsilon$ on $M$ by

$$
\varepsilon(X, Y)=\omega^{\prime}(\varphi(X), \varphi(Y)) .
$$

Set $\sigma=\varepsilon-\omega$. We shall prove that $\sigma \in \Gamma\left(\mathcal{S}_{(2)}^{2}\right)$ and that the couple $s=(\tilde{\delta}, \sigma)$ fulfils the equation

$$
D_{\omega} s-\frac{1}{2}[s, s]=0 .
$$

When $X \in \Gamma(F)$ then $\varphi(X) \in \Gamma\left(F^{\prime}\right)$ so $\varepsilon(X, Y)=0$ when $X \subseteq \Gamma(F)$. So $\varepsilon \subseteq \Gamma\left(\mathcal{S}_{(2)}^{2}\right)$. As $\omega$ is also a section of $\mathcal{S}_{(2)}^{2}$ then $\sigma \in \Gamma\left(\mathcal{S}_{(2)}^{2}\right)$. Equation (9) is equivalent to the two equations

$$
\begin{gathered}
D \tilde{\delta}-\frac{1}{2}[\tilde{\delta}, \tilde{\delta}]=0 \\
\tilde{\delta}(\omega+\sigma)-d \sigma=0 .
\end{gathered}
$$

The first one being satisfied, we have to prove only (11), As $\omega$ is closed (11) 
is equivalent to $(d-\tilde{\delta})(\omega+\sigma)=0$, that is $\delta(\omega+\sigma)=0$. Given the above local chart $\left(U, x^{u}, z^{a}\right)$ adapted to $\mathscr{F}$ let $\left\{\theta^{\lambda}\right\}$ be the dual basis of $\varphi_{\lambda}$. Since $\theta^{a} \in I_{F^{\prime}}$. then $\omega^{\prime}$ will be expressed

Then

$$
\omega^{\prime}=\Sigma \omega_{a b}^{\prime} \theta^{a} \wedge \theta^{b}
$$

$$
\varepsilon=\Sigma \omega_{a b}^{\prime} d z^{a} \wedge d z^{b}
$$

since $\varepsilon\left(\left(\partial / \partial z^{a}\right),\left(\partial / \partial z^{b}\right)\right)=\omega^{\prime}\left(\varphi_{a}, \varphi_{b}\right)=\omega_{a b}^{\prime}$. Let us write now the condition $d \omega^{\prime}=0$.

$$
0=d \omega^{\prime}=\sum \varphi_{\lambda}\left(\omega_{a b}^{\prime}\right) \theta^{\lambda} \wedge \theta^{a} \wedge \theta^{b}+\omega_{a b}^{\prime} d \theta^{a} \wedge \theta^{b}-\omega_{a b}^{\prime} \theta^{a} \wedge d \theta^{b} .
$$

But $d \theta^{\lambda}=-(1 / 2) \Sigma C_{\mu \nu}^{\lambda} \theta^{\mu} \wedge \theta^{\nu}$. So

$$
0=\Sigma\left(\varphi_{\lambda}\left(\omega_{\mu \nu}^{\prime}\right)-\frac{1}{2} \omega_{\rho \nu}^{\prime} C_{\lambda \mu}^{\rho_{\lambda}}+\frac{1}{2} \omega_{\lambda \rho}^{\prime} C_{\mu \nu}^{\rho}\right) \theta^{\lambda} \wedge \theta^{\mu} \wedge \theta^{\nu}
$$

where $\omega_{\mu \nu}^{\prime}=0$ when one of the two indices $\lambda$ or $\mu$ is an $u$ or an $\bar{a}$. On the other hand

$$
\begin{aligned}
\delta(\varepsilon)= & \sum \delta\left(\omega_{a b}^{\prime}\right) d z^{a} \wedge d z^{b}+\omega_{a b}^{\prime} \delta\left(d z^{a}\right) \wedge d z^{b}-\omega_{a b}^{\prime} d z^{a} \wedge \delta\left(d z^{b}\right) \\
= & (\text { by definition of } \delta) \\
=\sum \varphi_{\lambda}\left(\omega_{a b}^{\prime}\right) d x^{\lambda} \wedge d z^{a} \wedge d z^{b}- & \frac{1}{2} \omega_{a b}^{\prime} C_{\lambda \mu}^{a} d x^{\lambda} \wedge d x^{\mu} \wedge d z^{b} \\
& +\frac{1}{2} \omega_{a b}^{\prime} C_{\lambda \mu}^{b} d z^{a} \wedge d x^{\lambda} \wedge d x^{\mu} \\
=\Sigma\left(\varphi_{\lambda}\left(\omega_{\mu \nu}^{\prime}\right)-\frac{1}{2} \omega_{\rho \nu}^{\prime} C_{\lambda \mu}^{\rho}+\right. & \left.\frac{1}{2} \omega_{\lambda \rho}^{\prime} C_{\mu \nu}^{o}\right) d x^{\wedge} \wedge d x^{\mu} \wedge d x^{\nu}=0
\end{aligned}
$$

So $\delta(\omega+\sigma)=\delta \varepsilon=0$, proving (11),

Reciprocally, given a section $s=(\tilde{\delta}, \sigma)$ of $\Phi_{\omega}^{1}$ close enough to the zero section and fulfilling the integrability equation (9) we are going to associate a couple $\left(\mathscr{F}^{\prime}, \omega^{\prime}\right)$ to it, where $\mathscr{F}^{\prime}$ is a transversely holomorphic foliation with a transversely symplectic structure $\omega^{\prime}$.

Equation (9) is equivalent to (10) and (11). Let $\delta$ be the derivation $d-\tilde{\delta}$. Then $(10)$ is equivalent to $[\delta, \delta]=0$. Let $\varphi$ be the first component of $\delta$ ( $\varphi$ is a vector 1-form). Given a local chart $\left(U, x^{u}, z^{a}\right)$ adapted to $\mathscr{F}$ set $\varphi_{u}=\varphi\left(\partial / \partial x^{u}\right)$, $\varphi_{a}=\varphi\left(\partial / \partial z^{a}\right), \varphi_{\bar{a}}=\varphi\left(\partial / \partial \bar{z}^{a}\right)$. As $\varphi$ is close to the identity (since $\delta$ is close to $d$ ) $\left\{\varphi_{\lambda}\right\}$ is a basis at each point of $U$. Set $\left[\varphi_{\lambda}, \varphi_{\mu}\right]=\Sigma C_{\lambda \mu}^{\nu} \varphi_{\nu}$. Condition $[\delta, \delta]=0$ is equivalent to (8) (see [8]). Since $\delta \in \mathscr{D}^{1}$ one has $C_{\alpha \beta}^{a}=0$ when $\alpha, \beta \in\{u, \bar{\alpha}\}$. Set $F^{\prime}=\varphi(F)$. One has $\left[F^{\prime}, F^{\prime}\right] \subset F^{\prime}$. This integrability condition leads (by Newlander-Nirenberg theorem) to the existence of a transversely holomorphic foliation $\mathscr{F}^{\prime}$ whose associate bundle (in the sense of the introduction) is $F^{\prime}$. 
Condition $(10)$ is equivalent to $\delta(\omega+\sigma)=0$. Set $\gamma=\omega+\sigma$. Let $\omega^{\prime}$ the 2 -form given by

$$
\omega^{\prime}(X, Y)=\gamma\left(\varphi^{-1}(X), \varphi^{-1}(Y)\right) .
$$

From the condition $\delta(\gamma)=0$ one proves easily $i_{X} \omega^{\prime}=L_{X} \omega^{\prime}=0$ when $X \in \Gamma\left(F^{\prime}\right)$ and $d \omega^{\prime}=0$. So $\omega^{\prime}$ is a transversely symplectic structure on $\mathscr{F}^{\prime}$.

\subsection{The contact case}

Fix $(\mathscr{I}, \omega)$ a transversely holomorphic foliation $\mathscr{F}$ on a compact manifold $M$ with a transversely contact structure $\omega$ given by a family $\left\{\omega_{i}\right\}$ of 1 -forms at each adapted local chart $\left(U_{i}, x_{i}^{u}, z_{i}^{a}\right)$. Let us associate a section of $\Phi_{\omega}^{1}$ to each couple $\left(\mathscr{F}^{\prime}, \omega^{\prime}\right)$, where $\mathscr{F}^{\prime}$ is a transversely holomorphic foliation close enough to $\mathscr{F}$ and $\omega^{\prime}$ a transversely contact structure on $\mathscr{I}^{\prime}$ close enough to $\omega$. Let $\left\{U_{i}\right\}$ be a covering of $M$ such that each $U_{i}$ is the domain of an adapted local chart $\left(U_{i}, x_{i}^{u}, z_{i}^{a}\right)$ of $\mathscr{F}$ and domain of an adapted local chart $\left(U_{i}, y_{i}^{u}, \zeta_{i}^{a}\right)$ of $\mathcal{F}^{\prime}$. Suppose $\omega_{i}=e_{i j} \omega_{j}$ and $\omega_{i}^{\prime}=e_{i j}^{\prime} \omega_{j}^{\prime}$ on $U_{i} \cap U_{j}$, with $X\left(e_{i j}\right)=0$ if $X \in \Gamma(F)$ and $X^{\prime}\left(e_{i j}^{\prime}\right)=0$ if $X^{\prime} \in \Gamma\left(F^{\prime}\right)$. As in the symplectic case we associate to $\mathscr{F}^{\prime}$ a derivation $\delta$ fulfilling $[\delta, \delta]=0$. Set $\tilde{\delta}=d-\delta$. Then $[\delta, \delta]=0$ is equivalent to (10). We can find a family $\left\{\mu_{i}\right\}$ of functions on each $U_{i}$ such that $\mu_{i}-\mu_{j}=$ $\log \left(e_{i j}^{\prime} / e_{i j}\right)$ on $U_{i} \cap U_{j}$. Remark that as the $e_{i j}^{\prime}$ are close to $e_{i j}$ then the quotients $e_{i j}^{\prime} / e_{i j}$ are close to 1 , so we can choose a well-defined determination of the logaritm. Then the $\mu_{i}$ can be defined by

$$
\mu_{i}=\sum_{k} h_{k} \log \left(e_{i k}^{\prime} / e_{i k}\right),
$$

where $\left\{h_{k}\right\}$ is a partition of unity. Set $\gamma_{i}=(d-\tilde{\delta}) \mu_{i}=\delta \mu_{i}$. Let us prove that the couple $\left.\left(\tilde{\delta},\left[\gamma_{i}\right]\right\}\right)$ is an $E$-derivation with respect to te bundle $E$ with transition functions $\left\{e_{i j}\right\}$. We have

$$
\gamma_{i}-\gamma_{j}=\delta\left(\mu_{i}-\mu_{j}\right)=\delta\left(\log e_{i j}^{\prime}-\log e_{i j}\right) .
$$

But $\delta e_{i j}^{\prime}=\varphi \bar{\wedge} d e_{i j}^{\prime}=\varphi^{\lambda}\left(\partial e_{i j}^{\prime} / \partial z^{\lambda}\right)$, where $\varphi$ is the first component of $\delta$. As $\varphi(F)=$ $F^{\prime}$ we have $\left[\delta\left(e_{i j}^{\prime}\right)\right]=0$ because for $\alpha \in\{u, \bar{a}\}$ one has

$$
\sum \varphi_{\alpha}^{\lambda} \frac{\partial e_{i j}^{\prime}}{\partial z^{\lambda}}=\varphi_{\alpha}\left(e_{i j}^{\prime}\right)=0
$$

(since $X^{\prime}\left(e_{i j}^{\prime}\right)=0$ if $X^{\prime} \in \Gamma\left(F^{\prime}\right)$ ). So we have

$$
\left[\gamma_{i}\right]-\left[\gamma_{j}\right]=-\left[\delta\left(\log e_{i j}\right)\right]=\left[(d-\tilde{\delta})\left(\log e_{i j}\right)\right]=\left[\tilde{\delta}\left(\log e_{i j}\right)\right],
$$

because $d \log e_{i j} \in I_{F}$. So $\left(\left[\gamma_{i}\right]-\left[\gamma_{j}\right]\right) e_{i j}=\left[\tilde{\delta}\left(e_{i j}\right)\right]$ showing that $\left(\tilde{\delta},\left[\gamma_{i}\right]\right)$ is an $E$-derivation.

Set $\nu_{i}=\exp \mu_{i}$ and $c_{i}=\left(1 / \nu_{i}\right) \omega_{i}^{\prime}$. Let $\varepsilon_{i}$ be the 1 -form on $U_{i}$ given by 


$$
\varepsilon_{i}(X)=c_{i}(\varphi(X)) .
$$

Set $\sigma_{i}=\varepsilon_{i}-\omega_{i}$. One has $\sigma_{i}=e_{i j} \sigma_{j}$ on $U_{i} \cap U_{j}$. So $s=\left(\left(\tilde{\delta},\left\{\left[\gamma_{i}\right]\right\}\right),\left\{\left[\sigma_{i}\right]\right\}\right)$ is a section of $\Phi_{\omega}^{1}$. Let us prove that $s$ fulfils equation (9). One can see that (9) is equivalent to (10) and the two following equations

$$
\begin{gathered}
{\left[(d-\tilde{\delta}) \gamma_{i}\right]=0} \\
{\left[\tilde{\delta}\left(\omega_{i}+\sigma_{i}\right)-d \sigma_{i}\right]=\left[\gamma_{i} \wedge\left(\omega_{i}+\sigma_{i}\right)\right],}
\end{gathered}
$$

where the classes in (12) are classes modulo $I_{F}$ but the classes in (13) are classes modulo $I_{F} \wedge I_{F}$.

(12) is fulfilled because $\gamma_{i}=(d-\tilde{\delta}) \mu_{i}=\delta \mu_{i}$ and $\delta^{2}=0$ since $[\delta, \delta]=0$. Since $d \omega_{i} \in I_{F} \wedge I_{F}$ one has

$$
\left[\tilde{\delta}\left(\omega_{i}+\sigma_{i}\right)-d \sigma_{i}\right]=\left[(d-\delta)\left(\omega_{i}+\sigma_{i}\right)-d \sigma_{j}\right]=-\left[\delta\left(\omega_{i}+\sigma_{i}\right)\right]=-\left[\delta \varepsilon_{i}\right] .
$$

As in section 3.1 denote by $\left\{\theta^{\lambda}\right\}$ the dual basis of $\left\{\varphi_{\lambda}\right\}$, where $\varphi_{\lambda}=\varphi\left(\partial / \partial x^{\lambda}\right)$. If $\omega_{i}^{\prime}$ is expressed by

and

$$
\omega_{i}^{\prime}=\Sigma\left(\omega_{i}^{\prime}\right)_{a} \theta^{a}
$$

$$
\varepsilon_{i}=\Sigma\left(\varepsilon_{i}\right)_{a} d z^{a}
$$

one has, by definition of $\varepsilon_{i},\left(\varepsilon_{i}\right)_{a}=\left(\left(\omega_{i}^{\prime}\right)_{a} / \nu_{i}\right)$. So

$$
\varepsilon_{i}=\Sigma \frac{\left(\omega_{i}^{\prime}\right)_{a}}{\nu_{i}} d z^{a}
$$

But, as $\nu_{i}=\exp \mu_{i}, \delta\left(1 / \nu_{i}\right)=\delta\left(\exp \left(-\mu_{i}\right)\right)=-\exp \left(-\mu_{i}\right) \delta\left(\mu_{i}\right)=-\left(1 / \nu_{i}\right) \gamma_{i}$. So

$$
\left[\tilde{\delta}\left(\omega_{i}+\sigma_{i}\right)-d \sigma_{i}\right]=-\left[\delta \varepsilon_{i}\right]=\left[\gamma_{i} \wedge \varepsilon_{i}\right]-\frac{1}{\nu_{i}}\left[\delta\left(\Sigma\left(\omega_{i}^{\prime}\right)_{a} d z^{a}\right)\right]
$$

To prove (13) it suffices to prove that $\left[\delta\left(\Sigma\left(\omega_{i}^{\prime}\right)_{a} d z^{a}\right)\right]=0$. But

$$
\begin{aligned}
\delta\left(\sum\left(\omega_{i}^{\prime}\right)_{a} d z^{a}\right) & =\sum \varphi_{\lambda}\left(\left(\omega_{i}^{\prime}\right)_{a}\right) d x^{\lambda} \wedge d z^{a}+\left(\omega_{i}^{\prime}\right)_{a} \delta\left(d z^{a}\right) \\
& =\operatorname{by}(8) \\
& =\Sigma \varphi_{\lambda}\left(\left(\omega_{i}^{\prime}\right)_{a}\right) d x^{\lambda} \wedge d z^{a}-\frac{1}{2}\left(\omega_{i}^{\prime}\right)_{a} C_{\lambda \mu}^{a} d x^{\lambda} \wedge d x^{\mu}
\end{aligned}
$$

If we are only interested in classes modulo $I_{F} \wedge I_{F}$ we shall have

$$
\left[\delta\left(\Sigma\left(\omega_{i}^{\prime}\right)_{a} d z^{a}\right)\right]=\left[\Sigma \varphi_{\alpha}\left(\left(\omega_{i}^{\prime}\right)_{a}\right) d x^{\alpha} \wedge d z^{a}\right]-\left[\Sigma\left(\omega_{i}^{\prime}\right)_{a} C_{\alpha \mu}^{a} d x^{\alpha} \wedge d x^{\mu}\right],
$$

where $\alpha \in\{u, \bar{a}\}$. But as $\varphi_{\alpha}$ is a section of $F^{\prime}$ we have

$$
0=L_{\varphi_{\alpha}} \omega_{i}^{\prime}=\sum L_{\varphi_{\alpha}}\left(\left(\omega_{i}^{\prime}\right)_{a} \theta^{a}\right)=\Sigma \varphi_{\alpha}\left(\left(\omega_{i}^{\prime}\right)_{a}\right) \theta^{a}-C_{\alpha \mu}^{a}\left(\omega_{i}^{\prime}\right)_{a} \theta^{\mu} .
$$

Proving that

$$
\varphi_{\alpha}\left(\left(\omega_{i}^{\prime}\right)_{\mu}\right)-\Sigma C_{\alpha \mu}^{a}\left(\omega_{i}^{\prime}\right)_{a}=0
$$


(when $\mu \neq a$ then $\left(\omega_{i}^{\prime}\right)_{\mu}$ is zero). So $\left[\delta\left(\Sigma\left(\omega_{i}^{\prime}\right)_{a} d z^{a}\right]=0\right.$.

Reciprocally, given a section $s=\left(\left(\tilde{\delta},\left[\gamma_{i}\right]\right),\left[\sigma_{i}\right]\right)$ of $\Phi_{\omega}^{1}$ close enough to zero and fulfilling (9) one can associate a couple $\left(\mathscr{F}^{\prime}, \omega^{\prime}\right)$ to it close enough to $(\mathscr{F}, \omega)$. We do not give here this construction explicitily, but we remark that, as in the symplectic case, the Newlander-Nirenberg theorem must be used to associate a foliation $\mathscr{I}^{\prime}$ to $\tilde{\delta}$.

\section{Deformations of the structure parametrized by a non-reduced analytic space}

Given a (non-reduced) analytic space $S$ denote by $\Lambda_{S}^{t r}$ the pseudogroup of local $C^{\infty}$ automorphisms of $S \times \boldsymbol{R}^{p} \times \boldsymbol{C}^{q}$ of the form

$$
\left(s, x^{u}, z^{a}\right) \longmapsto\left(s, f^{v}(s, x, z), f^{b}(s, z)\right),
$$

where $u, v \in\{1, \cdots, p\}, a, b \in\{1, \cdots, q\}$ and the functions $f^{a}(s, z)$ are holomorphic. A family of transversely holomorphic foliations parametrized by $S$ is nothing but a topological space $\mathscr{X}$, a continuous map $\pi: \mathfrak{X} \rightarrow S$, a structure of $\Lambda_{S}^{t r}$-manifold on $\mathscr{X}$ given by an atlas $\left(V_{i}, \phi_{i}\right)$, where $V_{i} \subset \mathscr{X}$ is open and $\phi_{i}$ is a homeomorphism from $V_{i}$ onto an open subset of $S \times \boldsymbol{R}^{p} \times \boldsymbol{C}^{q}$, and a collection $\left\{\phi_{i j}\right\}$ of elements of $\Lambda_{S}^{t_{r}}$ verifying $\pi_{S} \circ \psi_{i}=\pi$ (where $\pi_{S}$ is the projection on S), $\phi_{i i}=\mathrm{id}, \phi_{i j^{\circ}} \phi_{j k}=\phi_{i k}$ and $\phi_{i}=\phi_{i j^{\circ}} \phi_{j}$, where in the last condition $\phi_{i j}$ is regarded only as a continuous map. For any $s \in S$ the fibre $M_{s}=\pi^{-1}(s)$ inherits a $\Lambda^{t r}$-structure, that is, we have $\left(M_{s}, \mathscr{F}_{s}\right)$ where $\mathscr{F}_{s}$ is a transversely holomorphic foliation on $M_{s}$.

Given $(M, \mathscr{I})$ a transversely holomorphic foliation on a compact manifold and an analytic space $S$ with a distinguished point $o \in S$, a family $\mathscr{X} \stackrel{\pi}{\rightarrow} S$ of transversely holomorphic foliations parametrized by $S$ is called a deformation of $(M, \mathscr{F})$ if there exists a $\Lambda^{t r}$-isomorphism $\iota:(M, \mathscr{F}) \rightarrow\left(M_{0}, \mathscr{F}_{0}\right)$. Such a deformation is denoted by $(\mathfrak{X}, \pi, S, o, \iota)$.

A complex vector bundle $\mathcal{E}$ of rank $k$ over $\mathscr{X}$ can be described as the object obtained by glueing together the open sets $V_{i} \times C^{k}$ by means of transformations of the form

$$
G_{i j}\left(s, x_{i}^{u}, z_{i}^{a}, t\right)=\left(s, \phi_{i j}^{v}\left(s, x_{j}^{u}, z_{j}^{b}\right), \phi_{i j}^{a}\left(s, z_{j}^{b}\right), g_{i j}\left(s, x_{j}^{u}, z_{j}^{b}\right) \cdot t\right),
$$

where the $\phi_{i j}$ are the coordinate changes of $\mathscr{X}$ and $g_{i j}$ are $\mathcal{C}^{\infty}$ functions with values in $\mathrm{GL}(k, C)$ depending holomorphically on $s$ and fulfilling the cocycle conditions $g_{i i}=1, g_{i k}=g_{i j} \cdot g_{j k}$. If $\mathcal{E} \rightarrow \mathfrak{X}$ is such a vector bundle, a section of $\mathcal{E}$ can be defined as a $\mathcal{C}^{\infty}$ morphism $\mathscr{X} \rightarrow \mathcal{E}$ which in each local chart is given by $(s, x, z) \mapsto(s, x, z, t(s, x, z))$. We shall denote by $\Gamma_{s}(\mathcal{E})$ the space of sections 
of $\mathcal{E}$.

When $k=p+2 q$ and $g_{i j}$ is the jacobian matrix

$$
\left(\begin{array}{ccc}
\frac{\partial \phi_{i j}^{v}}{\partial x_{j}^{u}} & \frac{\partial \phi_{i j}^{v}}{\partial z_{j}^{a}} & \frac{\partial \phi_{i j}^{v}}{\partial \bar{z}_{j}^{a}} \\
0 & \frac{\partial \phi_{i j}^{b}}{\partial z_{j}^{a}} & 0 \\
0 & 0 & \frac{\partial \bar{\phi}_{i j}^{b}}{\partial \bar{z}_{j}^{a}}
\end{array}\right)
$$

we obtain the bundle ${ }^{c} T \mathscr{X}$ called the complex tangent bundle of $\mathscr{X}$ along the fibres of $\mathfrak{X} \stackrel{\pi}{\rightarrow} S$. If $k=p+q$ and $g_{i j}$ is the jacobian matrix

$$
\left(\begin{array}{cc}
\frac{\partial \phi_{i j}^{v}}{\partial x_{i}^{u}} & \frac{\partial \phi_{i j}^{v}}{\partial \bar{z}_{j}^{a}} \\
0 & \frac{\partial \bar{\phi}_{i j}^{b}}{\partial \bar{z}_{j}^{a}}
\end{array}\right)
$$

whe obtain the bundle $F_{\mathfrak{X}} \rightarrow \mathfrak{X}$ associated to the natural transversely holomorphic foliation on $\mathscr{X}$.

We can define the space $A_{S}^{r}(\mathscr{X})$ of complex-valued $r$-forms on $\mathscr{X}$ as $\Gamma_{S}\left(\Lambda^{r}\left({ }^{c} T \mathscr{X}\right)^{*}\right)$. A natural exterior derivative $d: A_{S}^{r}(\mathscr{X}) \rightarrow A_{S}^{r+1}(\mathscr{X})$ is obtained derivating only with respect to the variables $x, z, \bar{z}$ but not with respect to the parameter $s$.

Given a transversely holomorphic foliation $(M, \mathscr{F})$ and a transversely symplectic structure $\omega$ on $\mathscr{F}$, a deformation of $(M, \mathscr{F}, \omega)$ is a couple $((\mathscr{X}, \pi, S, o, \iota)$, $\left.\omega_{s}\right)$, where $(\mathfrak{X}, \pi, S, o, \iota)$ is a deformation of $(M, \mathscr{I})$ and $\omega_{s} \in A_{S}^{2}(\mathscr{X})$ fulfilling $\iota^{*}\left(\omega_{0}\right)=\omega, i_{X} \omega_{s}=L_{X} \omega_{s}=0$ when $X \in \Gamma_{S}\left(F_{S}\right), \omega_{s}^{q^{\prime} \neq 0}$ and $d \omega_{s}=0 . \quad$ In an analogous way we can define the notion of deformation of $(M, \mathscr{F}, \omega)$ when $\omega$ is a transversely contact structure on $\mathscr{F}$.

Two deformations of $(M, \mathscr{F}, \omega),\left((\mathscr{X}, \pi, S, o, \iota), \omega_{s}\right)$ and $\left(\left(\mathscr{X}^{\prime}, \pi^{\prime}, S, o, \iota^{\prime}\right), \omega_{s}^{\prime}\right)$ parametrized by the same $(S, o)$, are called equivalent if there is an open neighbourhood $S^{\prime \prime}$ of $o$ in $S$ and an isomorphism $f:\left.\left.\mathscr{X}\right|_{S^{\prime \prime}} \rightarrow \mathscr{X}^{\prime}\right|_{S^{\prime \prime}}$ of $\Lambda_{S^{\prime \prime}}^{\text {tr }}$ manifolds over the identity of $S^{\prime \prime}$ such that $f^{*}\left(\omega_{s}^{\prime}\right)=\omega_{s}$ in the symplectic case and $f^{*}\left(\omega_{s}^{\prime}\right)=e \omega_{s}$ in the contact case, where $e$ is a transversely holomorphic function on $\left.\mathscr{X}\right|_{s "}$.

\section{Relation between deformations of $(M, \mathscr{F}, \omega)$ and families of sections of $\Phi_{\omega}^{1}$ fulfilling the integrability condition}

Let $(M, \mathscr{F}, \omega)$ be a transversely holomorphic foliation on a compact manifold $M$ with a transversely symplectic or contact structure $\omega$. It is not difficult to 
prove the existence of a vector bundle $E^{1}$ on $M$ whose space of sections $\Gamma\left(E^{1}\right)$ coincides with $\Gamma\left(\Phi_{\omega}^{1}\right)$, where $\Phi_{\omega}^{1}$ has been intruced in section 1 . Given an analytic space $S$ take the trivial family $\mathscr{X}=S \times M \rightarrow S$ and the trivial vector bundle $\mathcal{E}=S \times E^{1}$ over $\mathscr{X}$. We shall denote by $\Gamma_{S}\left(\Phi_{\omega}^{1}\right)$ the space $\Gamma_{S}(\mathcal{E})$ introduced in section 4. By putting parameters in the construction given in section 3, given a deformation $\left((\mathscr{X}, \pi, S, o, \iota), \omega_{s}\right)$ of $(M, \mathscr{T}, \omega)$ we can find an open neighbourhood $S^{\prime \prime}$ of $o$ in $S$ such that the construction of section 3 works in $S^{\prime \prime}$, that is, we are able to associate an element of $\Gamma_{S} \cdot\left(\Phi_{\omega}^{1}\right)$ fulfilling the integrability condition to the couple $\left(\left.\mathscr{X}\right|_{s}, \omega_{s}\right)$. Nevertheless, to associate (as in section 3 ) a family of deformations $\left(\mathscr{X}, \omega_{s}\right)$ over a suitable neighbourhood $S^{\prime \prime}$ of $o$ to each element of $\Gamma_{S}\left(\Phi_{\omega}^{1}\right)$ fulfilling the integrability condition we must use a Newlander-Nirenberg theorem with parameters (see section 3.) But such a theorem only works in the $C^{\infty}$ case. So we are lead to suppose that the initial manifold $M$ is $\mathcal{C}^{\omega}$ and that the initial foliation $\mathscr{T}$ on $M$ is also $\mathcal{C}^{\omega}$. With these assumptions we are able to associate to every element of ${ }^{\omega} \Gamma_{S}\left(E^{1}\right)$ fulfilling the integrability condition a family of deformations $\left(\mathscr{X}, \omega_{s}\right)$ of $(M, \mathscr{T}, \omega)$ over a suitable neighbourhood $S^{\prime \prime}$ of $o$. We may suppose without lost of generality that $(M, \mathscr{I})$ is $\mathcal{C}^{\omega}$ because any transversely analytic foliation is isotopic to a real analytic foliation $([9])$.

\section{Versality theorem}

Let $(M, \mathscr{T}, \omega)$ be a transversely holomorphic foliation $\mathscr{T}$ on a compact manifold $M$ endowed with a transversely holomorphic symplectic or contact structure $\omega$. Let $\left((\mathscr{X}, \pi, S, o, \iota), \omega_{s}\right)$ be a deformation of $(M, \mathscr{F}, \omega)$ parametrized by an analytic space $S$ with a distiguished point $o$ (we shall abbreviate such a deformation by $\left(\left.\mathscr{X}\right|_{s}, \omega_{s}\right)$ ). Given another analytic space $S^{\prime}$ with a distinguished point $o^{\prime}$ and a morphism $f: S^{\prime} \rightarrow S$ of analytic spaces with $f\left(o^{\prime}\right)=o$, let $\mathscr{X}_{f}$ be the fibre product $\mathfrak{X}_{f}=S^{\prime} \times{ }_{s} \mathscr{X}=\left\{\left(s^{\prime}, u\right) \in S^{\prime} \times \mathfrak{X} \mid f\left(s^{\prime}\right)=\pi(u)\right\}$. We shall have natural projections

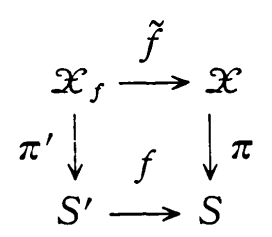

If $\left\{\left(V_{i}, \phi_{i}\right)\right\}$ is a $\Lambda_{S}^{t r}$-atlas of $\mathscr{X}$ with coordinate changes $\left\{\phi_{i j}\right\}$ we take the structure of $\Lambda_{S^{\prime}}^{t r}$-manifold on $\mathscr{X}_{f}$ given by the atlas $\left\{\left(\tilde{f}^{-1}\left(V_{i}\right), \varphi_{i}\right)\right\}$, where $\varphi_{i}$ : $\tilde{f}^{-1}\left(V_{i}\right) \rightarrow S^{\prime} \times\left(\boldsymbol{R}^{p} \times \boldsymbol{C}^{q}\right)$ is the map defined by $\varphi_{i}=\pi^{\prime} \times\left(\pi_{\boldsymbol{R}^{p} \times c^{q \circ}} \phi_{i} \circ \tilde{f}\right)$, and the coordinate changes $\phi_{i j}^{\prime}$ given by 


$$
\phi_{i j}^{\prime}:(s, x, z) \longrightarrow\left(s^{\prime}, \phi_{i j}^{u}(f(s), x, z), \phi_{i j}^{a}(f(s), z)\right) .
$$

Then $\tilde{f}$ restricted to $\pi^{\prime-1}(o)$ gives an isomorphism of $\Lambda^{t r}$-manifolds between $M_{o^{\prime}}=\pi^{\prime-1}\left(o^{\prime}\right)$ and $M_{o}=\pi^{-1}(o)$. Denote by $\iota^{\prime}$ the composition $(M, \mathscr{F}) \stackrel{\iota}{\rightarrow} M_{o} \stackrel{\tilde{f}^{-1}}{\rightarrow} M_{o^{\prime}}$. Then the couple $\left(\left(\mathfrak{X}_{f}, o^{\prime}, S^{\prime}, \iota^{\prime}\right), \tilde{f}^{*}\left(\omega_{s}\right)\right)$ is a deformation of $(M, \mathscr{F}, \omega)$ parametrized by $\left(S^{\prime}, o^{\prime}\right)$, called the inverse image of $\left((\mathfrak{X}, o, S, \iota), \omega_{s}\right)$.

Definition. A deformation $\left(\left.\mathscr{X}\right|_{s}, \omega_{s}\right)$ of $(M, \mathscr{F}, \omega)$ parametrized by $(S, o)$ is called versal if the two following conditions are fulfilled:

1. Given any other deformation $\left(\left.\mathfrak{X}^{\prime}\right|_{S^{\prime}}, \omega_{s^{\prime}}\right)$ of $(M, \mathscr{F}, \omega)$ parametrized by the analytic space $\left(S^{\prime}, o^{\prime}\right)$ there is a neighbourhood $S^{\prime \prime}$ of $o^{\prime}$ in $S^{\prime}$ and a morphism $f: S^{\prime \prime} \rightarrow S$ of analytic spaces, with $f\left(o^{\prime}\right)=o$, such that the inverse image $\left(\left.\mathscr{X}_{f}\right|_{s^{\prime \prime}}, f^{*}\left(\omega_{s}\right)\right)$ is equivalent to $\left(\left.\mathscr{X}^{\prime}\right|_{s^{\prime}}, \omega_{s^{\prime}}\right)$ (see the end of section 4 for the definition of equivalent).

2. The differential $d_{o^{\prime}} f$ at $o^{\prime}$ of the above morphism $f$ is unique (among those morphisms fulfilling condition 1 ).

THEOREM. (versality) Let $(M, \mathscr{F}, \omega)$ a transversely holomorphic foliation on a compact manifold endowed with a transversely holomorphic sympletic or contact structure. Then there exists a versal deformation $\left(\left.\mathfrak{X}\right|_{s}, \omega_{s}\right)$ of $(M, \mathscr{F}, \omega)$ parametrized by a pair $(S, o)$, where $o$ is the origin of the finite dimensional complex vector space $H^{1}\left(M, \Theta_{\omega}^{t r}\right)$ and $S$ is an analytic subspace of this cohomology space defined by an equation $f(s)=0$, where $f$ is a holomorphic map from a neighbourhood $U$ of the origin o of $H^{1}\left(M, \Theta_{\omega}^{t r}\right)$ into $H^{2}\left(M, \Theta_{\omega}^{t r}\right)$ whose jet of order 2 at $o$ is the quadratic form $s \rightarrow[s, s]$.

We shall not give here a detailed proof of this theorem. Simply we mention that the discussions in the preceding sections allows to translate here the standard construction of Douady [1] (see also [4]). We shall describe, however, how the versal family is built in order to use this construction in the computations of examples of the following sections.

Fix a large enough real positive number $r$ and denote by ${ }^{r} \Gamma\left(\Phi_{\omega}^{k}\right)$ the $r$ Sobolev's completion of the space $\Gamma\left(\Phi_{\omega}^{k}\right)$ of sections of the sheaf $\Phi_{\omega}^{k}$ introduced in section 1. Choose a real analytic riemannian metric on $M$ (by the reasoning at the end of section 5 we can suppose $M$ real analytic as well as $\mathscr{F})$. This metric induces real analytic scalar products in each ${ }^{r} \Gamma\left(\Phi_{\omega}^{k}\right)$. Let $D_{\omega}^{*}$ be the adjoint of $D_{\omega}:{ }^{r} \Gamma\left(\Phi_{\omega}^{k}\right) \rightarrow{ }^{r-1} \Gamma\left(\Phi_{\omega}^{k+1}\right)$ with respect to these products. Set $\Sigma=$ $\left\{s \in{ }^{r} \Gamma\left(\Phi_{\omega}^{1}\right)\right.$ such that $\left.D_{\omega}^{*}\left(D_{\omega} s-(1 / 2)[s, s]\right)=0\right\} . \quad \Sigma$ is a Banach submanifold of ${ }^{r} \Gamma\left(\Phi_{\omega}^{1}\right)$ in a neighbourhood of the origin whose tangent space $T_{0} \Sigma$ is ker $D_{\omega}$. 
Set $\tilde{H}=\Sigma \cap \operatorname{ker} D_{\omega}^{*}$. Then $\tilde{H}$ is a (finite dimensional) submanifold of $\Sigma$ in a neighbourhood of 0 with tangent space $T_{0} \tilde{H}=\operatorname{ker} D_{\omega} \cap \operatorname{ker} D_{\omega}^{*} \cong H^{1}\left(M, \Theta_{\omega}^{t r}\right)$. One can prove that $\tilde{H}$ can be defined alternatively as the set of those $s \in{ }^{r} \Gamma\left(\Phi_{\omega}^{1}\right)$ satisfying the elliptic equation

$$
D_{\omega}^{*}\left(D_{\omega} s-\frac{1}{2}[s, s]\right)+D_{\omega} D_{\omega}^{*} s=0 .
$$

This implies that the elements $s \in \tilde{H}$ belong to ${ }^{\infty} \Gamma\left(\Phi_{\omega}^{1}\right)$. Let $S$ be the analytic subspace of $\tilde{H}$ of those $s$ fulfilling the integrability equation $D_{\omega} s-(1 / 2)[s, s]=0$. In a neighbourhood of the origin each $s \in S$ defines (because of the integrability condition) a transversely holomorphic foliation $\mathscr{I}_{s}$ endowed with a tranversely holomorphic symplectic or contact structure $\omega_{s}$. Then $\left(\mathscr{I}_{s}, \omega_{s}\right)_{s \in S}$ is the versal family of deformations.

It is well known (see for example [9]) that an alternative decription of $\tilde{H}$ and $S$ is the following:

$$
\tilde{H}=\left\{s \in \Gamma\left(\Phi_{\omega}^{1}\right) \text { such that } s=H s+\frac{1}{2} D_{\omega}^{*} G[s, s]\right\}
$$

where $H s$ means the harmonic part of $s$ and $G$ denotes the Green's operator.

$$
S=\{s \in \tilde{H} \text { with } H[s, s]=0\}
$$

where $H[s, s]$ means the harmonic part of $[s, s]$.

We mention here the uniqueness (up to ismorphisms) of the versal space (see for example [4]) and the following extremely useful corollary of the versality theorem.

COROLLARY. (see [4]) Let $\left(\mathfrak{X}^{\prime} \mid s^{\prime}, \omega_{s^{\prime}}\right)$ be a deformation of $(M, \mathscr{F}, \omega)$ parametrized by $\left(S^{\prime}, o^{\prime}\right)$. If $S^{\prime}$ is smooth (that is, a neighbourhood of the origin of a complex vector space) and if the Kodaira-Spencer's map

$$
\rho: T_{o^{\prime}} S^{\prime} \longrightarrow H^{1}\left(M, \Theta_{\omega}^{t r}\right)
$$

is an isomorphism then $\left(\left.\mathfrak{X}^{\prime}\right|_{S^{\prime}}, \boldsymbol{\omega}_{s^{\prime}}\right)$ is versal.

The Kodaira-Spencer's map is defined as follows. If

$$
\phi_{i j}\left(s^{\prime}, x, z\right)=\left(s^{\prime}, \phi_{i j}^{u}\left(s^{\prime}, x, z\right), \phi_{i j}^{a}\left(s^{\prime}, z\right)\right)
$$

are the coordinate changes of $\mathfrak{X}^{\prime}$ and if $v \in T_{o^{\prime}} S^{\prime}$ then the vector fields

$$
X_{i j}=\Sigma v\left(\phi_{i j}^{a}\right) \frac{\partial}{\partial z_{i}^{a}}+\Sigma v\left(\phi_{i j}^{u}\right) \frac{\partial}{\partial x_{i}^{u}}
$$

give a 1-cocycle with values in $\Theta_{\omega}^{t r}$ whose cohomology class is $\rho(v)$. 


\section{The versal family of deformations of the examples given in the introduction}

\subsection{The Hopf fibration $S^{4 n+3} \rightarrow C P^{2 n+1}$}

The versal space of the transversely holomorphic foliation $\mathscr{I}$ on $S^{4 n+3}$ whose leaves are the fibres of the Hopf fibration is described in [3] and [4]. It is smooth, that is, a neighbourhood of the origin in the vector space $H^{1}\left(S^{4 n+3}, \Theta_{\omega}^{t r}\right)$ whose dimension is $(2 n+2)^{2}-1$. We are now interested in the versal space and the versal deformation of the same foliation with the additional transversely contact structure $\omega$ described in the introduction. Our first aim is the computation of $H^{1}\left(S^{4 n+3}, \Theta_{\omega}^{t r}\right)$. Denote by $\Theta$ the sheaf of germs of holomorphic vector fields on $\boldsymbol{C} \boldsymbol{P}^{2 n+1}$, by $\Theta_{\omega}$ the sheaf of germs of holomorphic vector fields $X$ on $\boldsymbol{C} \boldsymbol{P}^{2 n+1}$ fulfilling $L_{X} \omega_{i}=\lambda_{i} \omega_{i}$. Let $\mathcal{O}$ be the sheaf of germs of holomorphic functions on $\boldsymbol{C} \boldsymbol{P}^{2 n+1}$ and $\tilde{\Theta}$ the sheaf of germs of pairs $\left(X,\left\{\mu_{i}\right\}\right)$, where $X$ is a holomorphic vector field and the $\mu_{i}$ are holomorphic functions on each one of the $U_{i}$ fulfilling $\mu_{i}-\mu_{j}=X\left(e_{i j}\right) / e_{i j}$ on $U_{i} \cap U_{j}$. Finally, let $\Omega^{1}(E)$ be the sheaf of families of holomorphic 1-forms $\left\{\sigma_{i}\right\}$, each $\sigma_{i}$ being defined on $U_{i}$, and fulfilling $\sigma_{i}=e_{i j} \sigma_{j}$ on $U_{i} \cap U_{j}$. We have the following two exact sequences

$$
\begin{gathered}
0 \longrightarrow 0 \longrightarrow \tilde{\Theta} \longrightarrow \Theta \longrightarrow 0 \\
0 \longrightarrow \Theta_{\omega} \longrightarrow \tilde{\Theta} \longrightarrow \Omega^{1}(E) \longrightarrow 0
\end{gathered}
$$

where the projection $\tilde{\Theta} \rightarrow \Omega^{1}(E)$ is the map induced by

$$
\left(X,\left\{\mu_{i}\right\}\right) \longrightarrow\left\{L_{X} \omega_{i}-\mu_{i} \omega_{i}\right\} .
$$

Since $H^{i}\left(\boldsymbol{C P} \boldsymbol{P}^{2 n+1}, \Theta\right)=H^{i}\left(\boldsymbol{C} \boldsymbol{P}^{2 n+1}, \mathcal{O}\right)=0$ for $i>0$, we deduce from the first exact sequeuce that

$$
\operatorname{dim} H^{0}\left(\boldsymbol{C} \boldsymbol{P}^{2 n+1}, \tilde{\Theta}\right)=\operatorname{dim} H^{0}\left(\boldsymbol{C} \boldsymbol{P}^{2 n+1}, \Theta\right)+\operatorname{dim}\left(\boldsymbol{C} \boldsymbol{P}^{2 n+1}, \mathcal{O}\right)=(2 n+1)^{2}
$$

and $H^{1}\left(\boldsymbol{C} \boldsymbol{P}^{2 n+1}, \tilde{\Theta}\right)=0$. One can compute easily from the definitions the following dimensions:

$$
\begin{aligned}
& \operatorname{dim} H^{0}\left(\boldsymbol{C} \boldsymbol{P}^{2 n+1}, \Theta_{\omega}\right)=(2 n+2)^{2}-(1+2+\cdots+(2 n+1)) \\
& \operatorname{dim} H^{0}\left(\boldsymbol{C} \boldsymbol{P}^{2 n+1}, \Omega^{1}(E)\right)=1+2+\cdots+(2 n+1)
\end{aligned}
$$

Since, moreover, $H^{i}\left(\boldsymbol{C} \boldsymbol{P}^{2 n+1}, \Omega^{1}(E)\right)=0$ for $i>0$ we deduce from the second exact sequence the vanishing of $H^{1}\left(\boldsymbol{C} \boldsymbol{P}^{2 n+1}, \Theta_{\omega}\right)$ and $H^{2}\left(\boldsymbol{C} \boldsymbol{P}^{2 n+1}, \Theta_{\omega}\right)$. By virtue of the Leray's spectral sequence of the Hopf fibration $\pi: S^{4 n+3} \rightarrow \boldsymbol{C} \boldsymbol{P}^{2 n+1}$ we have the following exact sequence: 


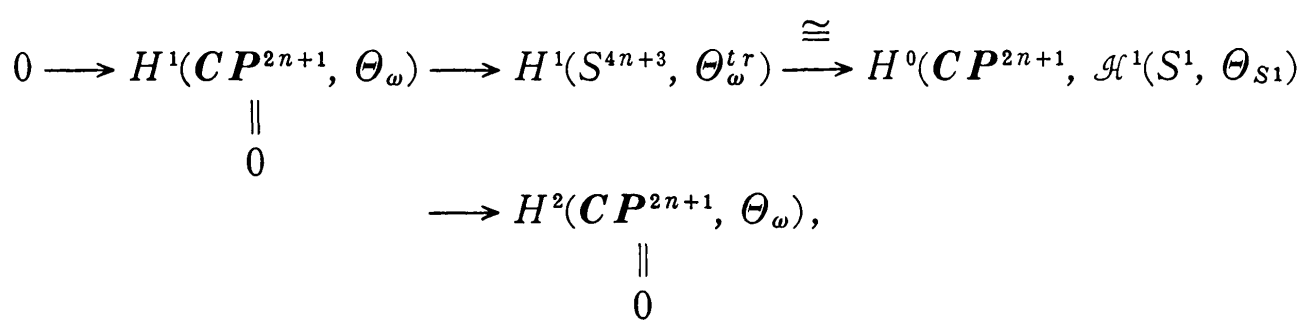

where $\mathscr{H}^{1}\left(S^{1}, \Theta_{S 1}\right)$ is the sheaf over $\boldsymbol{C} \boldsymbol{P}^{2 n+1}$ associating to $U$ the group $H^{1}\left(\pi^{-1}(U),\left.\Theta_{\omega}^{t r}\right|_{\pi^{-1}(U)}\right)$. When $U$ is small enough this group is isomorphic to $H^{0}\left(U,\left.\Theta_{\omega}\right|_{U}\right) \otimes H^{1}\left(S^{1}, \boldsymbol{R}\right)$.

So $\mathscr{H}^{1}\left(S^{1}, \Theta_{S 1}\right)$ is isomorphic to $\Theta_{\omega}$. Thus we have $H^{1}\left(S^{4 n+3}, \Theta_{\omega}^{t r}\right) \cong$ $H^{0}\left(\boldsymbol{C} \boldsymbol{P}^{2 n+1}, \Theta_{\omega}\right)$. That is

$$
\operatorname{dim} H^{1}\left(S^{4 n+3}, \Theta_{\omega}^{t r}\right)=(2 n+2)^{2}-(1+2+\cdots+(2 n+1)) .
$$

Following the ideas of [4] (the construction of the versal family for the transversely holomorphic foliation without contact structure) we want to built now a deformation of $\left(S^{4 n+3}, \mathscr{F}, \boldsymbol{\omega}\right)$ parametrized by $H^{0}\left(\boldsymbol{C} \boldsymbol{P}^{2 n+1}, \Theta_{\omega}\right)$. First of all remark that the fibres of the Hopf fibration are the intersection with $S^{4 n+3}$ of the (complex) integral curves of the radial vector field $\xi$ of $C^{2 n+2}$ :

$$
\xi=\sum z^{i} \frac{\partial}{\partial z^{i}} .
$$

Denote by $p$ the canonical projection $p: \boldsymbol{C}^{2 n+2} \rightarrow \boldsymbol{C} \boldsymbol{P}^{2 n+1}$. For each $\eta \in$ $H^{0}\left(\boldsymbol{C} \boldsymbol{P}^{2 n+1}, \Theta_{\omega}\right)$ take a holomorphic vector field $\tilde{\eta}$ on $\boldsymbol{C}^{2 n+2}$ whose projection by $p_{*}$ is $\eta$ and such that $[\tilde{\eta}, \xi]=0$. For $\eta$ small enough the (complex) integral curves of $\xi+\tilde{\eta}$ define a new foliation on $C^{2 n+2}-\{0\}$. Denote by $\mathscr{I}_{\eta}$ the foliation that it induces in $S^{4 n+3}$. We want to endow $\mathscr{I}_{\eta}$ with a transversely contact structure $\omega_{\eta}$.

The holomorphic vector fields $X$ on $\boldsymbol{C}^{2 n+2}$ conmuting with the radial vector field $\xi$ are linear. That is, of the form $X=\Sigma X^{i}\left(\partial / \partial z^{i}\right)$ with $X^{i}=\Sigma X_{j}^{i} X^{j}$, where $X_{j}^{i} \Subset \boldsymbol{C}$.

If $\alpha$ is now a 1 -form on $C^{2 n+2}$ of the form $\alpha=\sum \alpha_{i j} z^{i} d z^{j}$ with $\alpha_{i j} \in C$, the Lie derivative $L_{X} \alpha$ is given by

$$
L_{X} \alpha=\Sigma\left(X_{j}^{k} \alpha_{k i}+X_{i}^{k} \alpha_{j_{k}}\right) z^{j} d z^{i}
$$

We see, thus, that $L_{X} \alpha$ is also linear with coefficients $\left(L_{X} \alpha\right)_{i j} \in C$ given by

$$
\left(L_{X} \alpha\right)_{i j}=\Sigma\left(X_{j}^{k} \alpha_{k i}+X_{i}^{k} \alpha_{j_{k}}\right) .
$$

This suggests the use of the matrix notation. Denote also by $X$ the matrix $\left(X_{i}^{j}\right)$ and by $\alpha$ the matrix $\left(\alpha_{i j}\right)$. The above equality is written 


$$
L_{X} \alpha={ }^{t} X \alpha+\alpha X \text {. }
$$

If $\alpha$ is a linear form on $C^{2 n+2}$ and $X$ a linear vector field, condition $\alpha(X)=0$ is written in matrix notation

$$
{ }^{t} \alpha X+{ }^{t} X \alpha=0 .
$$

Let $\omega$ be the linear 1 -form on $C^{2 n+2}$

$$
\omega=\left(z^{1} d z^{2}-z^{2} d z^{1}\right)+\cdots+\left(z^{2 n+1} d z^{2 n+2}-z^{2 n+2} d z^{2 n+1}\right) .
$$

One has $\omega(\xi)=0$ and $L_{\xi} \omega=2 \omega$ (where $\xi$ is the radial vector field). For every $\eta \in H^{0}\left(\boldsymbol{C} \boldsymbol{P}^{2 n+1}, \Theta_{\omega}\right)$ (small enough) set $X=\xi+\tilde{\eta}$. We shall have $L_{X} \omega=K \omega$ (with $K$ constant). In matrix form we shall write this

$$
{ }^{t} X \omega+\omega X=K \omega .
$$

Find now a linear 1-form $\sigma$ fulfilling $\sigma(X)=0$ and $L_{X} \sigma=c \sigma$. In matrix notation this conditions will be written

$$
\left\{\begin{array}{c}
{ }^{t} X \sigma+\sigma X=c \sigma \\
{ }^{t} \sigma X+{ }^{t} X \sigma=0
\end{array}\right.
$$

Set $\mu={ }^{t} \sigma X$. These equations (in terms of $\mu$ ) become

$$
\left\{\begin{array}{l}
\mu X+{ }^{t} X \mu=c \mu \\
\mu+{ }^{t} \mu=0
\end{array}\right.
$$

which are fulfilled by $\mu=-\omega$ and $c=K$. So $\sigma={ }^{t} X^{-1} \omega$ fulfils the desired conditions (remark that $X$ has an inverse because $X=\xi+\tilde{\eta}$ with $\eta$ small).

At each small enough neighbourhood $U$ of a point of $C^{2 n+2}-\{0\}$ take a non-vanishing function $f_{U}$ fulfilling the equation $X\left(f_{U}\right)+c f_{U}=0$. Then $\alpha_{U}=f_{U} \sigma$ will be a 1 -form on $U$ such that $\alpha_{U}(X)=0$ and $L_{X} \alpha_{U}=0$. So it will be a basic 1-form on $U$ with respect to the foliation induced on $C^{2 n+2}$ by the vector field $X=\xi+\tilde{\eta}$. The restrictions of the $\alpha_{U}$ to the sphere $S^{4 n+3}$ (for those $U$ intersecting $S^{4 n+3}$ ) will give a transversely contact structure $\omega_{\eta}$ for the foliation $\mathscr{F}_{\eta}$. In this way we have a deformation $\left(S^{4 n+3}, \mathscr{F}_{\eta}, \omega_{\eta}\right)$ of the foliation $\left(S^{4 n+3}\right.$, $\mathscr{F}, \omega)$ parametrized by a neighbourhood of the origin of $H^{0}\left(\boldsymbol{C} \boldsymbol{P}^{2 n+1}, \Theta_{\omega}\right) \cong$ $H^{1}\left(S^{4 n+3}, \Theta_{\omega}^{t r}\right)$. The same argument of [4] applies now to show that the Kodaira-Spencer's map of this deformation is an isomorphism. So by virtue of the corollary of the theorem of versality, this deformation is versal.

\subsection{Other examples of transversely contact structures}

Let $M$ the projective cotangent bundle of $\boldsymbol{C} \boldsymbol{P}^{2 n+1}$ (that is, the projectiviza- 
tion of the cotangent bundle). The foliation given by the bundle $M \rightarrow C P^{2 n+1}$ admits a natural transversely contact structure (from the contact structure of $\left.\boldsymbol{C} \boldsymbol{P}^{2 n+1}\right)$. The Leray's spectral sequence of this bundle gives easily $H^{1}\left(M, \Theta_{\omega}^{t r}\right)$ $=0$, so that this structure is rigid.

The following example corresponds to a foliation with transversely contact structure whose versal family of deformations is parametrized by a nonsmooth analytic space. In the product manifold $M=T^{n} \boldsymbol{R} \times \boldsymbol{C} \boldsymbol{P}^{3}$ take the foliation whose leaves are the submanifolds $T^{n} \boldsymbol{R} \times\{p\}$ with the natural transversely contact structure induced by the contact structure $\boldsymbol{\omega}$ of $\boldsymbol{C} \boldsymbol{P}^{3}$. One has $\Theta_{\omega}^{t r}=$ $c \hat{\otimes} \Theta_{\omega}$, where $C$ denotes the constant sheaf with stalk $\boldsymbol{C}$ over $T^{n} \boldsymbol{R}$ and $\Theta_{\omega}$ is the sheaf over $\boldsymbol{C} \boldsymbol{P}^{3}$ of germs of holomorphic vector fieldr $X$ fulfilling $L_{X} \omega_{i}=$ $\lambda_{i} \omega_{i}$. As $H^{1}\left(\boldsymbol{C} \boldsymbol{P}^{3}, \Theta_{\omega}\right)=0$ one has

$$
H^{1}\left(M, \Theta_{\omega}^{t r}\right)=H^{1}\left(T^{n} \boldsymbol{R}, \boldsymbol{C}\right) \otimes H^{0}\left(\boldsymbol{C} \boldsymbol{P}^{3}, \Theta_{\omega}\right)
$$

wich is a space of complex dimension $10 n$.

In order to compute the versal space let us endow the space of sections of the sheaf $\Phi_{\omega}^{k}$ (of section 3.2) with a hermitian product in the following way. Take in $M$ the product of the Fubini's metric of $\boldsymbol{C} \boldsymbol{P}^{3}$ by the flat riemannian metric of $T^{n} \boldsymbol{R}$. If $\delta, \delta^{\prime} \in \mathscr{D}^{k}$ are expressed by the pairs of global vector forms $\delta=(\varphi, \eta), \delta^{\prime}=\left(\varphi^{\prime}, \eta^{\prime}\right)$ we define the hermitian product $\left\langle\delta, \delta^{\prime}\right\rangle$ by

$$
\left\langle\delta, \delta^{\prime}\right\rangle=\left\langle\varphi, \varphi^{\prime}\right\rangle+\left\langle\eta, \eta^{\prime}\right\rangle
$$

where $\left\langle\varphi, \varphi^{\prime}\right\rangle$ and $\left\langle\eta, \eta^{\prime}\right\rangle$ mean the usual products of vector forms. Let $E$ be the line bundle on $M$ defined by the transition functions $\left\{e_{i j}\right\}$. (Recall that $e_{i j}$ are the functions on $U_{i} \cap U_{j}$ such that $\left.\omega_{i}=e_{i j} \omega_{j}\right)$. Take a hermitian metric $h$ in the bundle $E$. If $s=\left(\delta,\left\{\gamma_{i}\right\},\left\{\sigma_{i}\right\}\right), s^{\prime}=\left(\delta^{\prime},\left\{\gamma_{i}^{\prime}\right\},\left\{\sigma_{i}^{\prime}\right\}\right)$ are sections of $\Phi_{\omega}^{k}$ (see section 3.2) we define the product

$$
\left\langle s, s^{\prime}\right\rangle=\left\langle\delta, \delta^{\prime}\right\rangle+\left\langle\delta\left(\omega_{i}\right)-\gamma_{i} \wedge \omega_{i}, \delta^{\prime}\left(\omega_{i}\right)-\gamma_{i}^{\prime} \wedge \omega_{i}\right\rangle_{h}+\left\langle\sigma_{i}, \sigma_{i}^{\prime}\right\rangle_{n}
$$

Remark that $\delta\left(\omega_{i}\right)-\gamma_{i} \wedge \omega_{i}$ is a (global) element of $S_{(1) F}^{k+1}(E)$, with the notations of section 3.2. Remark also that $\langle s, s\rangle=0$ implies $\delta=0, \sigma_{i}=0$ and $\gamma_{i} \wedge \omega_{i}$ $=0$. But as $\left\{\gamma_{i}\right\} \in S_{(1) F}^{k+1}(E)$ this implies $\gamma_{i}=0$. This proves that the hermitian product is non-degenerate.

Take a basis $\left\{X_{a}\right\}_{a=1, \ldots, 10}$ of $H^{0}\left(\boldsymbol{C} \boldsymbol{P}^{3}, \Theta_{\omega}\right)$ and denote by $\left\{d x^{u}\right\}_{u=1, \ldots, n}$ the 1-forms on $T^{n} \boldsymbol{R}$ induced by the canonical 1-forms $d x^{u}$ of $\boldsymbol{R}^{n}$. Denote by $\boldsymbol{\delta}_{a u}$ the element of $\mathscr{D}^{1}$ given by the pair of global vector forms $\left(d x^{u} \otimes X_{a}, 0\right)$. Let $\left(\lambda_{a}\right)_{i}$ be the holomorphic function on $U_{i} \subset \boldsymbol{C} \boldsymbol{P}^{3}$ such that $L_{X_{a}} \omega_{i}=\left(\lambda_{a}\right)_{i} \omega_{i}$. Denote by $s_{a u}$ the section of $\Phi_{\omega}^{1}$ given by 


$$
s_{a u}=\left(\delta_{a u},\left\{\left(\lambda_{a}\right)_{i} d x^{u}\right\},\{0\}\right)
$$

One can prove that each $s_{a u}$ is harmonic. The bracket $\left[s_{a u}, s_{b v}\right]$ is given by

$$
\left[s_{a u}, s_{b v}\right]=\left(\left[\delta_{a u}, \delta_{b v}\right],\left\{\left(X_{a}\left(\lambda_{b}\right)_{i}-X_{b}\left(\lambda_{a}\right)_{i}\right) d x^{u} \wedge d x^{v}\right\},\{0\}\right)
$$

where $\left[\delta_{a u}, \delta_{b v}\right]$ is the derivation given by the pair of global vector forms $\left(\left(d x^{u} \otimes d x^{v}\right) \otimes\left[X_{a}, X_{b}\right], 0\right)$. One sees that the brackets $\left[s_{a u}, s_{b v}\right]$ are also harmonic. According to section 6 the versal space will be (in this case) the analytic subspace of $C^{10 n}$ defined by the equation $[s, s]=0$, where $s$ is expressed in the basis $\left\{s_{a u}\right\}$ of harmonic elements of $\Gamma\left(\Phi_{\omega}^{1}\right)$ by $s=\sum k^{a u} s_{a u}$ with $k^{a u}$ complex numbers.

If $n=1$ then $u=v=1$ and in this case the brackets $\left[s_{a u}, s_{b v}\right]=0$ so that (only in this case) the versal space is smooth.

\subsection{The suspension of $T^{2 n} C$ by an element of $\operatorname{Sp}(2 n, Z)$}

Let $T^{2 n} \boldsymbol{C}$ be the complex torus $\boldsymbol{C}^{2 n} /(\boldsymbol{Z}+i \boldsymbol{Z})^{2 n}$. Given $A \in \mathrm{Sp}(2 n, \boldsymbol{Z})$ we take the quotient manifold $M$ of $\boldsymbol{R} \times T^{2 n} \boldsymbol{C}$ by the equivalence relation identifying $(t, z)$ with $(t+1, A(z))$. Take the transversely holomorphic foliation $\mathscr{F}$ on $M$ whose leaves are induced by the lines $R \times\{z\}$. As $A$ preserves the symplectic 2-form $\omega=d z^{1} \wedge d z^{2}+\cdots+d z^{2 n-1} \wedge d z^{2 n}$ on $T^{2 n} C$ then $\mathscr{F}$ is transversely symplectic. We want to construct the versal family of deformations of $(M, \mathscr{F}, \omega)$.

We have a natural projection $\pi: M \rightarrow S^{1}$ whose fibres are transverse to $\mathscr{F}$. We can then use the Leray's spectral sequence of $\pi$ to compute the cohomology space $H^{1}\left(M, \Theta_{\omega}^{t r}\right)$. We shall have the following exact sequence

$$
\begin{aligned}
0 \longrightarrow H^{1}\left(S^{1}, \pi\left(\Theta_{\omega}^{t r}\right)\right) & \longrightarrow H^{1}\left(M, \Theta_{\omega}^{t r}\right) \longrightarrow H^{0}\left(S^{1}, \mathcal{H}^{1}\left(T^{2 n} C, \Theta_{\omega}^{t r}\right)\right) \\
& \longrightarrow H^{2}\left(S^{1}, \pi\left(\Theta_{\omega}^{t r}\right)\right)
\end{aligned}
$$

As $S^{1}$ can be covered by two contractible open sets with contractible intersections and as $\pi\left(\Theta_{\omega}^{t r}\right)$ is a locally constant sheaf we obtain easily that $H\left(S^{1}, \pi\left(\Theta_{\omega}^{t r}\right)\right)=0$.

Let us compute $H^{1}\left(S^{1}, \pi\left(\Theta_{\omega}^{t r}\right)\right)$. Set $\left.U_{1}=\right] 0,1\left[\subset \boldsymbol{R}, U_{2}=\right] 1 / 2,3 / 2\left[\subset \boldsymbol{R}, V_{1}=\right.$ $p\left(U_{1} \times T^{2 n} \boldsymbol{C}\right), V_{2}=p\left(U_{2} \times T^{2 n} \boldsymbol{C}\right)$, where $p: \boldsymbol{R} \times T^{2 n} \boldsymbol{C} \rightarrow M$ is the canonical projection. On each $V_{i}$ we can take coordinates

$$
\begin{aligned}
\varphi_{i}: V_{i} & \longrightarrow U_{i} \times T^{2 n} C \\
x & \longrightarrow\left(t_{i}, z_{i}^{1}, \cdots, z_{i}^{2 n}\right)
\end{aligned}
$$

with $p\left(t_{i}, z_{i}^{1}, \cdots, z_{i}^{2 n}\right)=x$. In $V_{1} \cap V_{2}$ we shall have 


$$
\left.\left.\left.\begin{array}{l}
t_{2}=t_{1}+1 \\
z_{2}=A\left(z_{1}\right)
\end{array}\right\} \text { if } t_{1} \in\right] 0,1 / 2\left[\begin{array}{l}
t_{2}=t_{1} \\
z_{2}=z_{1}
\end{array}\right\} \text { if } t_{1} \in\right] 1 / 2,1[
$$

Let $\tilde{U}_{1}, \tilde{U}_{2}$ be the open sets in $S^{1}$ given by $U_{1}$ and $U_{2}$. In the Čech cohomology of the covering $\left\{\tilde{U}_{1}, \tilde{U}_{2}\right\}$ with values in the sheaf $\pi\left(\Theta_{\omega}^{t r}\right)$ the 0 -cohains are the couples $\left(s_{1}, s_{2}\right)$ with

$$
\begin{aligned}
& s_{1}=\Sigma \alpha^{a} \frac{\partial}{\partial z_{1}^{a}} \\
& s_{2}=\Sigma \beta^{a} \frac{\partial}{\partial z_{2}^{a}}
\end{aligned}
$$

where $\alpha^{a}$ and $\beta^{a}$ are constants. The 1-cochains are sections $s_{12}$ of $\pi\left(\Theta_{\omega}^{t r}\right)$ on $\tilde{U}_{1} \cap \tilde{U}_{2}$ given by

$$
s_{12}(t)= \begin{cases}\sum x^{a} \frac{\partial}{\partial z_{1}^{a}} & \text { when } t \in] 0,1 / 2[ \\ \sum y^{a} \frac{\partial}{\partial z_{2}^{a}} & \text { when } t \in] 1 / 2,1[\end{cases}
$$

with $x^{a}, y^{a}$ constants. As $\left(\partial\left(s_{1}, s_{2}\right)\right)_{12}=s_{2}-s_{1}$ we deduce easily that the degree 1 cohomology is the quotient of the global holomorphic vector fields on $T^{2 n} C$ by the image of the morphism $I-A_{*}$, where $I$ is the identity and $A_{*}$ is the morphism induced by $A$ on the vector fields, because the cocycle $s_{12}$ given by $X_{1}=\Sigma X_{1}^{a}\left(\partial / \partial z_{1}^{a}\right)$ when $\left.t \in\right] 0,1 / 2\left[\right.$ and by $X_{2}=\sum X_{2}^{a}\left(\partial / \partial z_{2}^{a}\right)$ when $\left.t \in\right] 1 / 2,1[$ defines the same cohomology class that the cocycle given by $\Sigma\left(X_{1}^{a}-X_{2}^{a}\right)\left(\partial / \partial z_{1}^{a}\right)$ when $t \in] 0,1 / 2[$ and by 0 when $t \in] 1 / 2,1\left[\right.$ (since the difference is $\partial\left(0, X_{2}\right)$ ). So $H^{1}\left(S^{1}, \pi\left(\Theta_{\omega}^{t r}\right)\right)$ is isomorphic to the vector space of holomorphic vector fields on $T^{2 n} \boldsymbol{C}$ invariant by $A_{*}$.

Let us compute now $H^{0}\left(S^{1}, \mathscr{H}^{1}\left(T^{2 n} \boldsymbol{C}, \Theta_{\omega}^{t r}\right)\right)$. Denote by $\Theta_{\omega}$ the sheaf of germs of holomorphic vector fields $X$ on $T^{2 n} C$ such that $L_{X} \omega=0$. First of all compute $H^{1}\left(T^{2 n} C, \Theta_{\omega}\right)$. The condition $L_{X} \omega$ is equivalent to the fact that the 1 -form $\sigma_{X}=X^{2} d z^{1}+X^{1} d z^{2}+\cdots$ is closed (that is, locally exact). This gives a natural exact sequence of sheaves

$$
0 \longrightarrow \mathrm{C} \longrightarrow \mathrm{O} \longrightarrow \Theta_{\omega} \longrightarrow 0
$$

where $\mathcal{C}$ is the constant sheaf with stalk $C$ and $\mathcal{O}$ the sheaf of germs of holomorphic functions. From the associated cohomology sequence (by using the fact that the morphism $H^{2}(\mathcal{C}) \rightarrow H^{2}(\mathcal{O})$ is the projection of the space of 2 -forms into the space of $(0,2)$-forms $)$ we deduce that

$$
\operatorname{dim} H^{1}\left(T^{2 n} C, \Theta_{\omega}\right)=(2 n)^{2}+\left(\begin{array}{c}
2 n \\
2
\end{array}\right) .
$$


In terms of the resolution of $\Theta_{\omega}$ given in 1.1 (corresponding to the foliation on $T^{2 n} \boldsymbol{C}$ whose leaves are the points) a basis of $H^{1}\left(T^{2 n} \boldsymbol{C}, \Theta_{\omega}\right)$ can be given by the sections of $\Phi_{\omega}^{1}$ given by $s_{a}^{b}=\left(\delta_{a}^{b}, 0\right), a, b \in\{1, \cdots, 2 n\}, s_{a b}^{\prime}=\left(0, d z^{a} \wedge d z^{b}\right), a$, $b \in\{1, \cdots, 2 n\}$ with $a<b$, where $\delta_{a}^{b}$ is the 1 -derivation given by the couple of global vector forms $\left(d \bar{z}^{\alpha} \otimes\left(\partial / \partial z^{b}\right), 0\right)$.

Let us give now a basis of $H^{1}\left(M, \Theta_{\omega}^{t r}\right)$ (which is the direct sum of $H^{1}\left(S^{1}\right.$, $\left.\pi\left(\Theta_{\omega}^{t r}\right)\right)$ and $H^{0}\left(S^{1}, \mathcal{H}^{1}\left(T^{2 n} C, \Theta_{\omega}^{t r}\right)\right)$. If $X$ is a holomorphic vector field on $T^{2 n} \boldsymbol{C}$ invariant by $A$, let $\delta_{X}$ be the 1-derivation on $M$ given by the couple of global vector forms ( $d t \otimes X, 0)$, where $t$ is the coordinate of $S^{1}$. Let $s_{X}$ be the (closed) section of $\Phi_{\omega}^{1}$ given by $s_{X}=\left(\delta_{X}, 0\right)$. If $\varphi$ and $\varepsilon$ are matrices fulfilling $\varphi A=A \varphi$ and ${ }^{t} A \varepsilon A=\varepsilon$, denote by $s_{\varphi}$ and $s_{\varepsilon}$ the (closed) sections of $\Phi_{\omega}^{1}$ given by the couples $s_{\varphi}=\left(\sum \varphi_{b}^{a} \delta_{b}^{a}, 0\right), s_{\varepsilon}=\left(0, \sum \varepsilon_{a b} d z^{a} \wedge d z^{b}\right)$. Let $X_{k}, \varphi_{l}, \varepsilon_{m}$ be bases of the spaces of vector fields on $T^{2 n} C$ invariant by $A$, of the matrices $\varphi$ fulfilling $\varphi A=$ $A \varphi$ and of the matrices $\varepsilon$ such that ${ }^{t} A \varepsilon A=\varepsilon$. Then the classes of the sections $s_{X}, s_{\varphi_{l}}, s_{\varepsilon_{m}}$ are a basis of $H^{1}\left(M, \Theta_{\omega}^{t r}\right)$.

As the brackets of each couple of linear combinations of these sections vanish then the following family of sections of $\Phi_{\omega}^{1}$ parametrized by $H^{1}\left(M, \Theta_{\omega}^{t r}\right)$

$$
\begin{aligned}
H^{1}\left(M, \Theta_{\omega}^{t r}\right) & \longrightarrow \Gamma\left(\Phi_{\omega}^{1}\right) \\
\Sigma\left(C^{k} s_{X_{k}}+C^{l} s_{\varphi_{l}}+C^{m} s_{\varepsilon_{m}}\right) & \longrightarrow \Sigma\left(C^{k} s_{X_{k}}+C^{l} s_{\varphi_{l}}+C^{m} s_{\varepsilon_{m}}\right)
\end{aligned}
$$

is an integrable family. As the Kodaira-Spencer's morphism of this family is the identity then this family is vesal by the corollary of the versality theorem.

\section{Examples of transversely symplectic foliations with versal families of deformations parametrized by non-smooth analytic spaces}

Let $T^{2 n} \boldsymbol{C}$ be the complex torus $\boldsymbol{C}^{2 n} /(\boldsymbol{Z}+i \boldsymbol{Z})^{2 n}$ with its symplectic form $\omega=d z^{1} \wedge d z^{2}+\cdots+d z^{2 n-1} \wedge d z^{2 n}$. Let $\pi: M \rightarrow T^{2 n} C$ be a principal $S^{1}$-bundle over $T^{2 n} \boldsymbol{C}$. We shall suppose that the Euler class $e(M)$ of $M$ belongs to $H^{1,1}\left(T^{2 n} \boldsymbol{C}\right.$, $C)$. This is equivalent to the existence of a holomorphic line bundle $L \rightarrow T^{2 n} C$ with a hermitian metric $h$ such that the principal $U(1)$-bundle of unitary vectors of $L$ is isomorphic to the bundle $M \rightarrow T^{2 n} C$.

Let $\mathscr{F}$ be the foliation on $M$ whose leaves are the fibres of $\pi$. $\mathscr{F}$ is endowed with the transversely symplectic structure given by $\omega$. Our purpose is to describe the versal family of deformations of $(M, \mathscr{F}, \omega)$. Take a connection $\rho^{\prime}$ on $M$ ( $\rho^{\prime}$ is a global 1 -form taking imaginary values on real vectors). We can choose $\rho^{\prime}$ such that its curvature $d \rho^{\prime}$ is harmonic in $T^{2 n} C$. Set $\rho=$ $(1 / 2 \pi i) \rho^{\prime}$. Then $\Omega=d \rho \in H^{1,1}\left(T^{2 n} C\right)$ is the harmonic representative of $e(M)$. 
We have $f_{S_{1}} \rho=1$, where $f_{S^{1}}$ means the integral along the fibres.

From the Leray's spectral sequence of $M \rightarrow T^{2 n} C$ we have

$$
0 \longrightarrow H^{1}\left(T^{2 n} \boldsymbol{C}, \Theta_{\omega}\right) \longrightarrow H^{1}\left(M, \Theta_{\omega}^{t r}\right) \longrightarrow H^{0}\left(T^{2 n} C, \Theta_{\omega}\right) \stackrel{\Delta}{\longrightarrow} H^{2}\left(T^{2 n} C, \Theta_{\omega}^{t r}\right)
$$

To compute $H^{1}\left(M, \Theta_{\omega}^{t r}\right)$ from this sequence we need the dimension of the kernel of $\Delta$. To this end we prefer to describe the above exact sequence from a differential point of view instead of obtaining it from the Leray's spectral sequence. Let us begin by introducing resolutions of the sheaves $\Theta_{\omega}^{t r}$ and $\Theta_{\omega}$ different from the general resolution introduced in section 1.1 . If $z^{1}, \cdots, z^{2 n}$ are the coordinates in (an open set) $T^{2 n} C$ induced by the canonical coordinates of $C^{2 n}$ then $\left\{d z^{a}\right\}$ are global 1-forms and $\left\{d z^{a}, d \bar{z}, \rho\right\}$ is a basis of the space of vector 1 -forms at each point of $M$. Let $\left\{Z_{a}, \bar{Z}_{a},(\partial / \partial t)\right\}$ its dual basis. Then

$$
Z_{a}=\frac{\partial}{\partial z^{a}}-\rho\left(\frac{\partial}{\partial z^{a}}\right) \frac{\partial}{\partial t}
$$

is the horizontal lift of $\left(\partial / \partial z^{a}\right)$. Every vector form $\varphi$ is expresed in the basis $\left\{Z_{a}, \bar{Z}_{a},(\partial / \partial t)\right\}$ by

$$
\varphi=\Sigma \varphi^{a} Z_{a}+\Sigma \varphi^{\bar{a}} Z_{\bar{a}}+\varphi^{t} \frac{\partial}{\partial t}
$$

where $\varphi^{a}, \varphi^{\bar{a}}$ and $\varphi^{t}$ are ordinary forms. We shall say that $\varphi$ is horizontal of type $(1,0)$ if $\varphi^{\bar{a}}=\varphi^{t}=0$. Denote by $\mathscr{D}^{\prime p}$ the space of degree $p$ derivations given by couples of global vector forms $(\varphi, \eta)$ with $\varphi$ and $\eta$ horizontal of type $(1,0)$. Remark that if $\delta \in \mathscr{D}^{\prime p}$ then $D \delta \in \mathscr{D}^{p+1}$. Let $\Phi_{\omega}^{\prime p}$ be the sheaf of germs of elements of $\mathscr{D}^{p} \oplus S_{(2)}^{p+1}$ when $p \neq 0$. Let $\Phi_{\omega}^{\prime 0}$ be the sheaf of germs of elements of $\mathscr{D}^{\prime 0}$. We shall have the following fine resolution of $\Theta_{\omega}^{t r}$ :

$$
0 \longrightarrow \Theta_{\omega}^{t r} \longrightarrow \Phi_{\omega}^{\prime 0} \stackrel{D_{\omega}}{\longrightarrow} \Phi_{\omega}^{\prime 1} \stackrel{D_{\omega}}{\longrightarrow} \cdots
$$

where $D_{\omega}$ is the differential introduced in section 1.1. When we take the trivial foliation in $T^{2 n} C$ whose leaves are the points we obtain a resolution of $\Theta_{\omega}$

$$
0 \longrightarrow \Theta_{\omega} \longrightarrow \Phi_{\omega}^{\prime 0} \stackrel{D_{\omega}}{\longrightarrow} \Phi_{\omega}^{\prime 1} \longrightarrow
$$

(we denote by $\Phi_{\omega}^{\prime *}$ the resolution of $\Theta_{\omega}$ as the resolution of $\Theta_{\omega}^{t r}$ ).

We define the integration along the leaves

$$
f_{S^{1}}: \Gamma\left(M, \Phi_{\omega}^{\prime p}\right) \longrightarrow \Gamma\left(T^{2 n} C, \Phi_{\omega}^{\prime p-1}\right)
$$

in the following way. If $\alpha=(\delta, \sigma) \in \Gamma\left(M, \Phi_{\omega}^{\prime p}\right)$ with $\delta=(\varphi, \eta) \in \mathscr{D}^{\prime p}, \varphi=\Sigma \varphi^{a} Z_{a}$, 
$\eta=\Sigma \eta^{a} Z_{a}$, then $f_{S^{1}} \alpha=\left(f_{S^{1}} \delta, f_{S^{1}} \sigma\right)$ where $f_{S^{1}} \delta$ is the degree $(p-1)$ derivation given by tha pair of global forms

$$
\left(\Sigma\left(f_{S^{1}} \varphi^{a}\right) \otimes \frac{\partial}{\partial z^{a}}, \Sigma\left(f_{S 1} \eta^{a}\right) \otimes \frac{\partial}{\partial z^{a}}\right)
$$

The morphism $\int_{S 1}: \Gamma\left(M, \Phi_{\omega}^{\prime p}\right) \rightarrow \Gamma\left(T^{2 n} C, \Phi_{\omega}^{\prime p-1}\right)$ is onto. Denote by $K^{p}$ its kernel. We have the following exact sequence of differential complexes

$$
0 \longrightarrow K^{p} \longrightarrow \Gamma\left(M, \Phi_{\omega}^{\prime p}\right) \longrightarrow \Gamma\left(T^{2 n} \boldsymbol{C}, \Phi_{\omega}^{\prime p-1}\right) \longrightarrow 0
$$

leading to the following cohomology sequence

$$
\cdots \longrightarrow H^{p}\left(K^{*}\right) \longrightarrow H^{p}\left(M, \Theta_{\omega}^{t r}\right) \longrightarrow H^{p-1}\left(T^{2 n} \boldsymbol{C}, \Theta_{\omega}\right) \stackrel{\Delta^{\prime}}{\longrightarrow} H^{p+1}\left(K^{*}\right) \longrightarrow \cdots
$$

If $\delta=(\varphi, \eta) \in \mathscr{D}_{T^{2 n} c}^{\prime p}, \varphi=\Sigma \varphi^{a}\left(\partial / \partial z^{a}\right), \eta=\Sigma \eta^{a}\left(\partial / \partial z^{a}\right)$, denote by $\lambda(\delta)$ the element of $\mathscr{D}_{M}^{\prime p}$ given by the pair $\left(\Sigma \pi^{*}\left(\varphi^{a}\right) Z_{a}, \Sigma \pi^{*}\left(\eta^{a}\right) Z_{a}\right)$. Denote also by $\lambda$ the map

$$
\begin{gathered}
\lambda: \Gamma\left(T^{2 n} C, \Phi_{\omega}^{\prime p}\right) \longrightarrow K^{p} \subset \Gamma\left(M, \Phi_{\omega}^{\prime p}\right) \\
(\delta, \sigma) \longrightarrow\left(\lambda(\delta), \pi^{*} \sigma\right)
\end{gathered}
$$

$\lambda$ is a morphism of complexes which induces an isomorphism in cohomology. The morphism $\Delta=\lambda_{*}^{-1} \circ \Delta^{\prime}$

$$
\Delta: H^{0}\left(T^{2 n} \boldsymbol{C}, \Theta_{\omega}\right) \longrightarrow H^{2}\left(M, \Theta_{\omega}^{t r}\right)
$$

is given by $X \rightarrow$ class of $\left(0,-\Omega \wedge i_{X} \omega\right)$. So $X$ will belong to ker $\Delta$ if $(0,-\Omega \wedge$ $\left.i_{X} \omega\right)=D_{\omega}(\delta, \sigma)=(d \delta, \delta(\omega)-d \sigma)$. As $D \delta=0$ then $\delta$ is a pair $(\varphi, 0)$ and $\delta(\omega)=$ $-d(\varphi \bar{\wedge} \omega)$. So $\Omega \wedge i_{X} \omega=d(\varphi \bar{\wedge} \omega-\sigma)$. As $\Omega$ is harmonic this is equivalent to $\Omega \wedge i_{X} \omega=0$. So

ker $\Delta=\left\{X\right.$ holomorphic vector fields on $T^{2 n} C$ fulfilling $\left.\Omega \wedge i_{X} \omega=0\right\}$

From the exact sequence

$$
0 \longrightarrow H^{1}\left(T^{2 n} \boldsymbol{C}, \Theta_{\omega}\right) \longrightarrow H^{1}\left(M, \Theta_{\omega}^{t r}\right) \longrightarrow \operatorname{ker} \Delta \longrightarrow 0
$$

we deduce

$$
\operatorname{dim} H^{1}\left(M, \Theta_{\omega}^{t r}\right)=(2 n)^{2}+\left(\begin{array}{c}
2 n \\
2
\end{array}\right)+\operatorname{dim} \operatorname{ker} \Delta
$$

To compute the versal family of deformations of $(M, \mathscr{F}, \omega)$ let us define hermitian products in $\Gamma\left(M, \Phi_{\omega}^{\prime p}\right)$. We define a riemannian metric $g$ on $M$ by

$$
\begin{aligned}
& g\left(Z_{a}, \bar{Z}_{b}\right)=\delta_{a b} \\
& g\left(\frac{\partial}{\partial t}, \frac{\partial}{\partial t}\right)=1
\end{aligned}
$$




$$
g\left(Z_{a}, \frac{\partial}{\partial t}\right)=g\left(\bar{Z}_{a}, \frac{\partial}{\partial t}\right)=g\left(Z_{a}, Z_{b}\right)=0
$$

If $\varphi$ and $\phi$ are horizontal vector forms of type $(1,0), \varphi=\Sigma \varphi^{a} Z_{a}, \phi=\Sigma \psi^{a} Z_{a}$, we define their hermitian product $\langle\varphi, \phi\rangle$ by

$$
\langle\varphi, \phi\rangle=\int_{M} \Sigma \varphi^{a} \wedge \overline{* \phi^{b}} g\left(Z_{a}, \bar{Z}_{b}\right)
$$

where $*$ means the Hodge's operator associated to $g$. We define a hermitian product $\langle$,$\rangle in \mathscr{D}^{p}$ in the following way. If $\delta_{1}, \delta_{2} \in \mathscr{D}^{\prime p}$ are represented by couples of global forms $\delta_{1}=\left(\varphi_{1}, \eta_{1}\right), \delta_{2}=\left(\varphi_{2}, \eta_{2}\right)$ then

$$
\left\langle\delta_{1}, \delta_{2}\right\rangle=\left\langle\varphi_{1}, \varphi_{2}\right\rangle+\left\langle\eta_{1}, \eta_{2}\right\rangle \text {. }
$$

Finally we define a hermitian product $\langle$, $\rangle$ in $\Gamma\left(M, \Phi_{\omega}^{\prime p}\right)$ in the following way. If $\alpha_{1}, \alpha_{2} \in \Gamma\left(M, \Phi_{\omega}^{\prime p}\right)$ are represented by couples $\alpha_{1}=\left(\delta_{1}, \sigma_{1}\right), \alpha_{2}=\left(\delta_{2}, \sigma_{2}\right)$ where $\delta_{1}=\left(\varphi_{1}, \eta_{1}\right), \delta_{2}=\left(\varphi_{2}, \eta_{2}\right)$ then

$$
\left\langle\alpha_{1}, \alpha_{2}\right\rangle=\left\langle\delta_{1}, \delta_{2}\right\rangle+\left\langle\sigma_{1}-(-1)^{p} \varphi_{1} \bar{\wedge} \omega, \sigma_{2}-(-1)^{p} \varphi_{2} \bar{\wedge} \omega\right\rangle
$$

Now denote by $\varphi_{a b}$ the vector 1 -form $d \bar{z}^{a} \otimes Z_{b}, a, b \in\{1, \cdots, 2 n\}$. Let $\delta_{a b}$ be the (closed) derivation given by the couple of global forms $\delta_{a b}=\left(\varphi_{a b}, 0\right)$. Let $\alpha_{a b}$ be the section of $\Phi_{\omega}^{\prime 1}$ given by the couple $\alpha_{a b}=\left(\delta_{a b}, 0\right)$. Denote by $\beta_{c d}$ the element of $\Gamma\left(M, \Phi_{\omega}^{\prime 1}\right)$ given by the couple $\beta_{c d}=\left(0, d z^{c} \wedge d z^{d}\right)$ with $c<d$ and $c$, $d \in\{1, \cdots, 2 n\}$. Take now a basis $\left\{X_{i}\right\}$ of $\operatorname{ker} \Delta \subset H^{0}\left(T^{2 n} C, \Theta_{\omega}\right)$ (vector fields fulfilling $\left.\Omega \wedge i_{X_{i}} \omega=0\right)$. Denote by $\tilde{X}_{i}$ the horizontal lift of $X_{i}$. Set $\varphi_{i}=\rho \otimes \tilde{X}_{i}$. Let $\delta_{i}$ be the (closed) derivation given by the pair $\delta_{i}=\left(\varphi_{i}, 0\right)$. Let $\gamma_{i}$ be the section of $\Phi_{\omega}^{\prime 1}$ given by the couple $\gamma_{i}=\left(\delta_{i}, 0\right)$. It is not difficult to see that $\left\{\alpha_{a b}, \beta_{c d}, \gamma_{i}\right\}$ is a basis of the space $H^{1}$ of harmonic elements of $\Gamma\left(M, \Phi_{\omega}^{\prime 1}\right)$. An easy computation shows that

$$
\begin{gathered}
{\left[\alpha_{a b}, \alpha_{c d}\right]=0} \\
{\left[\alpha_{a b}, \beta_{c d}\right]=0} \\
{\left[\alpha_{a b}, \gamma_{i}\right]=\left(\left(d \bar{z}^{a} \wedge i_{\partial / \partial z} \Omega\right) \otimes \tilde{X}_{i}, 0\right)} \\
{\left[\beta_{a b}, \beta_{c d}\right]=0} \\
{\left[\beta_{a b}, \gamma_{i}\right]=\left(0, \Omega \wedge i_{\tilde{X}_{i}} \sigma_{a b}\right)} \\
{\left[\gamma_{i}, \gamma_{j}\right]=\left(\left(\left(\rho \wedge i_{Z_{i}} \Omega\right) \otimes Z_{j}+\left(\rho \wedge i_{Z_{j}}\right) \otimes Z_{i}, 0\right), 0\right)}
\end{gathered}
$$

All these brackets are also harmonic sections of $\Phi_{\omega}^{\prime 2}$.

As the Kuranishi's space $S$ is defined by

$$
S=\{s \in \tilde{H} \text { such that } H[s, s]=0\}
$$


and $\tilde{H}$ is the space

$$
\tilde{H}=\left\{\alpha \in \Gamma\left(M, \Phi_{\omega}^{\prime 1}\right) \text { with } \alpha=H \alpha+\frac{1}{2} D_{\omega}^{*}[\alpha, \alpha]\right\}
$$

we see that (in this case) $S=\left\{s \in H^{1}\right.$ such that $\left.[s, s]=0\right\}$. If $s$ is expressed by

$$
s=\sum s^{a b} \alpha_{a b}+s^{l m} \beta_{l m}+s^{i} \gamma_{i}
$$

then the equation $[s, s]=0$ is equivalent to

$$
\sum 2 s^{a b} s^{j}\left[\alpha_{a b}, \gamma_{i}\right]+2 s^{l m} s^{j}\left[\beta_{l m}, \gamma_{i}\right]+s^{i} s^{j}\left[\gamma_{i}, \gamma_{j}\right]=0
$$

We see that $S$ contains always the submanifold given by the equations $s^{j}=0$ for $j \in\{1, \cdots, \operatorname{dim} \operatorname{ker} \Delta\}$, corresponding to the deformations of the complex and the symplectic structure of $T^{2 n} \boldsymbol{C}$. When $\operatorname{dim} \operatorname{ker} \Delta=0$ then $S$ is smooth. But, in general, $S$ is not.

For example, suppose that $n=1$ and that the Euler class of $M \rightarrow T^{2 n} C$ is

$$
\Omega=A d z^{1} \wedge d \bar{z}^{1}+B d z^{2} \wedge d \bar{z}^{1}-\bar{B} d z^{1} \wedge d \bar{z}^{2}+C d z^{2} \wedge d \bar{z}^{2}
$$

with $A$ and $C$ complex numbers with vanishing real part and $B$ any complex number. In this case ker $\Delta$ is the space of vector fields $X=X^{1}\left(\partial / \partial z^{1}\right)+X^{2}\left(\partial / \partial z^{2}\right)$ with $X^{1}$ and $X^{2}$ complex numbers fulfilling

$$
\left\{\begin{array}{l}
A X^{1}+B X^{2}=0 \\
\bar{B} X^{1}-C X^{2}=0
\end{array}\right.
$$

When $A C+B \bar{B} \neq 0$ then $\operatorname{ker} \Delta=\{0\}$. But when $A C+B \bar{B}=0$ then $\operatorname{ker} \Delta$ is not trivial. Suppose, for example, $A \neq 0$. Then $\operatorname{ker} \Delta$ is the 1-dimensional space generated by the vector field $X=(B / A)\left(\partial / \partial z^{1}\right)-\left(\partial / \partial z^{2}\right)$. In this case the dimension of $H^{1}\left(M, \Theta_{\omega}^{t r}\right)$ is 6 and $S$ is given by the equations

$$
\left.\begin{array}{r}
s^{\prime \prime} s^{\prime}=0 \\
s^{\prime \prime}\left(B s^{11}+(B \bar{B} / A) s^{12}+A s^{21}+B s^{22}\right)=0
\end{array}\right\}
$$

where $s^{11}, s^{12}, s^{21}, s^{22}, s^{\prime}$ and $s^{\prime \prime}$ are the coordinates associated to the basis $\left\{\alpha_{11}\right.$, $\left.\alpha_{12}, \alpha_{21}, \alpha_{22}, \beta, \gamma\right\}$ described above.

\section{References}

[1] A. Douady, Le problème des modules pour les variétés analytiques complexes. Séminaire Bourbaki 17éme année (1964/65) n² 277.

[2] T. Duchamp and M. Kalka, Deformation theory for holomorphic foliations, J. Differential Geometry, 14 (1979), 317-337.

[3] T. Duchamp and M. Kalka, Holomorphic foliations and deformations of the Hopf foliation, Pacific Journal of Mathematics, Vol. 112 No. 1 (1984), 69-81. 
[4] J. Girbau, A. Haefliger and D. Sundararaman, On deformations of transvesely holomorphic foliations, Journal für die reine und angew. Math. 345 (1983), 122-147.

[5] X. Gómez-Mont, Transversal holomorphic structures, J. Differential Geometry 15 (1980), 161-165.

[6] S. Kobayashi, Remarks on complex contact manifolds, Proc. Amer. Math. Soc. 10 (1959), 164-167.

[7] K. Kodaira, On deformations of some complex pseudo-group structures, Ann. of Math. 71 (1960), 224-302.

[8] K. Kodaira and D.C. Spencer, Multifoliate structures, Ann. of Math. 74 (1961), 52-100.

[9] M. Kuranishi, Deformations of compact complex manifolds, Montréal (1971).

[10] A. Haefliger, Structures feuilletées et cohomologie dans un faisceau de grupoïdes, Comm. Math. Helv. 32 (1958), 248-329. 